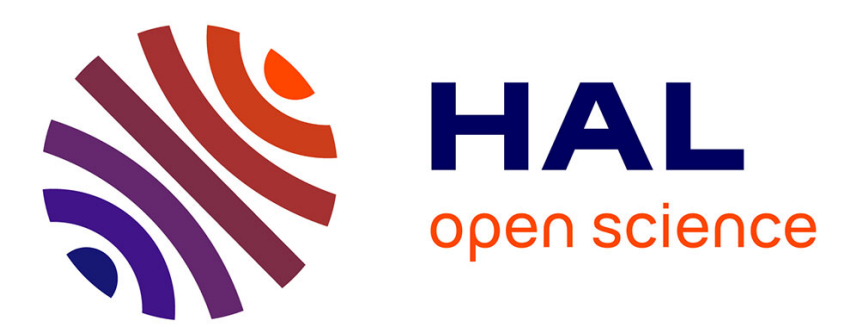

\title{
Numerical predictions of turbulence/cascade-interaction noise using computational aeroacoustics with a stochastic model
}

Cyril Polacsek, Vincent Clair, Thomas Le Garrec, Gabriel Reboul, Marc C. Jacob

\section{To cite this version:}

Cyril Polacsek, Vincent Clair, Thomas Le Garrec, Gabriel Reboul, Marc C. Jacob. Numerical predictions of turbulence/cascade-interaction noise using computational aeroacoustics with a stochastic model. AIAA Journal, 2015, 53 (12), pp.3551-3566. 10.2514/1.J053896 . hal-01296921

\section{HAL Id: hal-01296921 https://hal.science/hal-01296921}

Submitted on 17 Apr 2019

HAL is a multi-disciplinary open access archive for the deposit and dissemination of scientific research documents, whether they are published or not. The documents may come from teaching and research institutions in France or abroad, or from public or private research centers.
L'archive ouverte pluridisciplinaire HAL, est destinée au dépôt et à la diffusion de documents scientifiques de niveau recherche, publiés ou non, émanant des établissements d'enseignement et de recherche français ou étrangers, des laboratoires publics ou privés. 


\title{
Numerical predictions of turbulence-cascade interaction
}

\section{noise using CAA with a stochastic model}

\author{
C. Polacsek ${ }^{1}$, V. Clair ${ }^{2}$, T. Le Garrec ${ }^{1}$, G. Reboul ${ }^{1}$, \\ ONERA-The French Aerospace Lab, 92322 Châtillon, France \\ M. C. Jacob ${ }^{3}$ \\ Université Claude Bernard Lyon I, Laboratoire de Mécanique des Fluides et d'Acoustique, \\ CNRS-UMR 5509, 69622 Villeurbanne Cedex, France
}

Turbulent flow interactions with the outlet guide vanes are known to mainly contribute to broadband noise emission of aeroengines at approach conditions. This paper presents a 3D CAA hybrid method aiming at simulating the aeroacoustic response of an annular cascade impacted by a prescribed homogeneous isotropic turbulent flow. It is based on a time-domain Euler solver coupled to a synthetic turbulence model implemented in the code by means of a suited inflow boundary condition. The fluctuating pressure over the airfoil surface provided by CAA is used as an input to a Ffowcs-Williams and Hawkings integral method to calculate the radiated sound field. Euler computations are first validated against an academic CAA benchmark in the case of an harmonic gust interacting with an annular flat plate cascade. Then, simulations are applied to turbulence-cascade interactions for annular configurations, in uniform and swirling mean flows, and numerical results in terms of sound power spectra in the outlet duct are compared to semi-analytical and numerical solutions, and to an available experiment.

\footnotetext{
${ }^{1}$ Research Engineer, CFD and Aeroacoustics Department, cyril.polacsek@onera.fr.

2 (PhD at Onera) Research Fellow, Institute of Sound and Vibration Research, V.J.Clair@ soton.ac.uk.

${ }_{3}^{3}$ Assistant Professor, Université Claude Bernard and Polytech Lyon LMFA, marc.jacob@ec-lyon.fr.
} 


\section{Nomenclature}

$$
\begin{aligned}
& B \quad=\text { blade number } \\
& L \quad=\text { span } \\
& T_{u} \quad=\text { turbulence intensity } \\
& U_{0}, U_{x} \quad=\text { uniform, axial mean flow velocity } \\
& V \quad=\text { vane number } \\
& c \quad=\text { chord } \\
& f \quad=\text { frequency } \\
& k=\text { wave number } \\
& m, m_{g} \quad=\text { acoustic spinning mode order, gust spinning mode order } \\
& p, p \quad=\quad \text { static pressure, } \text { undisturbed static pressure } \\
& u^{\prime} \quad=\text { velocity disturbance } \\
& (x, r, \theta)=\text { cylindrical coordinates in the annular duct } \\
& \Delta k, \Delta f \quad=\text { wave number and frequency spacing } \\
& \chi \quad=\text { stagger angle } \\
& \left(k_{x}, k_{r}\right)=2 \text {-wavenumbers turbulent energy spectrum } \\
& \Lambda=\text { integral length scale } \\
& \varphi \quad=\text { random phase } \\
& \rho, \quad=\text { density, undisturbed density } \\
& \omega=\text { angular frequency } \\
& (,, r)=\text { local coordinates attached to the vane }
\end{aligned}
$$




\section{Introduction}

Turbulent wakes generated by turbofan blades and interacting with the outlet guide vanes are known to mainly contribute to broadband noise emission of aeroengines at approach conditions. Analytical approaches, such as Amiet's ${ }^{1}$ isolated airfoil or Hanson's ${ }^{2}$ cascade models can be adopted to estimate the noise generated by turbulent flows impacting thin airfoils, but they are limited by the flat-plate assumptions. Despite some recent attempts ${ }^{3-5}$, reliable rotor-stator turbulent interaction sources are still out of reach of common CFD solvers based on LES or DES approaches. These simulations are generally restricted to a radial strip and to a single vane channel by enforcing periodicity conditions, and it should be more considered for capturing the 3D turbulent wake behind an isolated rotor blade as investigated for example in Ref. [6]. Recently, the Lattice-Boltzmann Method (LBM) has been firstly applied to turbomachinery noise problems with an impressive direct acoustic simulation performed by $\mathrm{EXA}^{7}$ on the NASA Glenn Advanced Noise Control Fan model, including the full rotor-stator stage and the wind tunnel walls. The LBM technique appears to be a quite promising way to face present limitations in terms of current CPU capabilities.

Another approach ${ }^{8}$ based on a CAA/Euler hybrid methodology coupled to a synthetic turbulence inflow can also be considered, as investigated by recent studies ${ }^{9-11}$, and is the object of the present paper. Here, we suggest to numerically assess the aerodynamic response of annular grids impacted by a prescribed turbulent velocity field, instead of using airfoil or cascade flat plate response models adopted in the semi-analytical prediction tools. The turbulent wake generation from the rotor blades, devoted to CFD, is discarded in the present study.

The method is described in the first part of the paper, focusing on the way of modeling and injecting a synthetic turbulent flow (in terms of solenoidal velocity disturbances) in a CAA Euler code developed at Onera $^{12,13}$, respectively using a prescribed isotropic homogeneous TKE (Turbulence Kinetic Energy) spectrum expanded into spatial Fourier modes, and a suited BC (Boundary Condition) proposed by $\operatorname{Tam}^{14}$. 
In the second part, the numerical simulations are validated against an academic benchmark related to a 3D annular cascade impacted by a swirling harmonic gust in a uniform axial mean flow proposed by Namba \& Schulten ${ }^{15}$. The CAA results are compared to the semi-analytical solutions addressed by Namba $^{16}$ and Schulten ${ }^{17}$. For this test case, the acoustic response of the cascade (in-duct sound field) is directly assessed by the CAA.

Then the method is applied to the simulation of turbulence-cascade interaction noise on two selected configurations:

- A turbulence-annular cascade interaction in a uniform axial mean flow, related to a laboratory experiment performed in the anechoic open jet wind tunnel of Ecole Centrale de Lyon $^{18}$ and chosen as a fundamental case in the framework of a recent workshop on broadband turbofan noise prediction ${ }^{19}$;

- A turbulence-annular cascade interaction in a swirling mean flow, related to a benchmark proposed by Atassi \& Vinogradov ${ }^{20}$, with a reference solution issued from the frequency-domain linearized Euler code developed by Atassi et al. ${ }^{21}$.

For these more complex cases involving broadband sources, the CAA domain is limited to a single vane channel and azimuthal dependency of synthetic turbulent inflow is discarded, which permits to use periodic boundary conditions in the angular direction. With these restrictions, direct acoustic field cannot be provided anymore by the CAA. The sound radiation in the outlet duct is obtained by means of a FWH (Ffowcs-Williams and Hawkings) formulation (restricted to the loading noise term) generalized by Goldstein $^{22}$ for annular ducts, using the CAA pressure fluctuations along vane surface as input data, and assuming a fully uniform flow in the propagation (even for the swirling mean flow case). The present numerical predictions of in-duct Power Spectrum Density (PSD) in the outlet duct (downstream of the cascade) are compared to available measurements and analytical solutions too, issued from Amiet theory extended to ducted fans ${ }^{23,24}$ and advanced 3D lifting surface calculations ${ }^{25}$. The Amiet-based formulation developed by Reboul ${ }^{24}$ with proper expressions used in Onera updated code are addressed in an Appendix. Thus, challenging issues related to cascade and swirling mean flow effects on sound radiation, as well as 
the reliability of the simplifications adopted in the present CAA when simulating the incoming turbulent flow are both discussed.

\section{Hybrid method based on stochastic model coupled to CAA}

The simulations are performed using the ONERA code $s A b r i n A . v O^{12,13}$ solving the non-linearized or linearized Euler equations (NLEE or LEE, respectively) in the time domain with a perturbation form that consists in a splitting of the conservative variables into a mean flow and a disturbance field. The spatial derivatives are computed using a 6th order finite difference scheme and a 10th order explicit filter is applied to remove high frequency oscillations. This allows to avoid numerical dispersion and dissipation effects for grids satisfying at least 10-points-per-wavelength condition. The time evolution is achieved using a 3rd order Runge-Kutta scheme. In order to perform rotor-stator interaction problems through CAA linearized Euler calculations, efficient numerical BC (asymptotic solutions of the LEE) derived by $\operatorname{Tam}^{14}$ have been implemented in the code ${ }^{8}$ to allow velocity perturbations to be imposed at the inflow boundary. Although Tam's boundary conditions are initially written in 2D polar coordinates, a more suited form extended to spherical coordinates ${ }^{26}$ is used for ducted cascade calculations. As done in Ref. [26], a sponge zone (over-filtering), combined to a mesh stretching, is applied too at the exit of the CAA domain in order to allow both hydrodynamic and acoustic outgoing waves to leave the domain without generating spurious numerical reflections. A literature review and investigation of advanced synthetic turbulence generation models devoted to turbofan applications have been recently studied by $\mathrm{Sescu}^{27}$. The proposed stochastic model is the most simple and is similar to Kraichnan's theory ${ }^{28}$. As proposed by Kraichnan and also adopted in Ref. [29], it is based on a Fourier-mode decomposition of the incoming turbulent wake modeled by an HIT (Homogeneous Isotropic Turbulence) energy spectrum, but restricted here to the upwash velocity component (normal to the airfoil assimilated to a flat plate) by analogy with Amiet's theory. Such an approach aims to reproduce the statistical energy of prescribed TKE spectrum in the frequency domain without trying to capture any space-time correlation scales, which is different to time-domain RPM (Random-Particule Mesh) methods extensively used by Ewert ${ }^{30}$. 
However, 2D and 3D turbulence-airfoil simulations studied in Ref. [8] were found to be as much accurate as those (only 2D) reported in Ref. [9] using RPM approach. Moreover, in order to limit the size of the CAA domain and CPU cost, and following the approach of Casper and Farassat ${ }^{31}$, the synthetic turbulence is described here by a 2-wave numbers spectrum (the 3D HIT spectrum is integrated over the azimuthal wave numbers), with a spatial distribution over streamwise and spanwise directions. Thus, neglecting the azimuthal wave number dependency of the TKE spectrum, the 2-wave numbers spectrum approach suggested by Clair ${ }^{8}$ for simulating turbulence-airfoil problem is re-considered here for present annular cascade configurations. These restrictions are discussed in the applications presented in section IV. Hence, the incoming gusts (tangential component $u$ only), in the case of a purely axial mean flow and annular cascade with zero stagger angle, can be written as:

$$
u^{\prime}(x, r, t)=2 \sum_{i=1}^{N} \sum_{j=M}^{M} \sqrt{{ }_{u} u\left(k_{x, i}, k_{r, j}\right) k_{x} k_{r}} \cos \left(k_{x, i} x+k_{r, j} r \quad{ }_{i} t+{ }_{i, j}\right)
$$

In Eq. (1), the mode amplitude is fitted by a Von-Karman or Liepmann energy spectrum $\left(k_{x}, k_{r}\right)$, defined by two parameters: the turbulence intensity $\left(T_{u}\right)$ and the integral length scale $(\Lambda)$. Considering a frozen turbulence, the turbulent structures are assumed to be convected through the undisturbed upstream flow (mean velocity $U_{0}$ ), so that the angular frequency $\omega$ is related to the streamwise (axial) wave number $k_{x}$ (aligned to the vane chord) by: $k_{x}=\omega / U_{x} . \varphi_{i, j}$ is a random phase chosen between 0 and $2 \pi$ associated to each mode $(i, j)$. The synthetic turbulent field so obtained is solenoidal (divergence free), but if it is used with the full Euler equations, it is not solution of the radial momentum equation due to a nonlinear term. Thus, standard LEE are solved in the CAA to prevent the generation ofspurious oscillations.

As already discussed in the introduction, although acoustic propagation might be directly assessed by CAA as done in section III related to spinning harmonic gust-cascade interactions, restrictions considered in Eq. (1) aiming at limiting the CAA domain to a single vane channel (using angular periodicity conditions) do not permit to capture the radiated sound field. It is practically obtained by a coupling to a 
FWH formulation (loading noise term) using a Green's function valid for annular ducts and uniform axial mean flow ${ }^{23,24}$, implemented in a Fortran90 code (FanNoise) developed by Polacsek and Reboul.

\section{Validations on academic NASA benchmark cases}

Firstly, our numerical method has been validated against $3^{\text {rd }}$ CAA benchmark cases proposed by $\mathrm{NASA}^{15}$, devoted to the simulation of a swirling harmonic gust interacting with an annular cascade ( $V=24$ flat plates with chord $c=1 \mathrm{~m})$ in an axial uniform mean flow $(M=0.5)$. This case was also successfully simulated by Hixon et al. in Ref. [32]. The inflow velocity disturbances using cylindrical coordinates $(x, r, \theta)$ are defined as:

$$
\left\{\begin{array}{l}
u_{r}^{\prime}(r,, x, t)=0 \\
u^{\prime}(r,, x, t)=A \cos \left(k_{x} x+m_{g}+k_{r}\left(\begin{array}{ll}
r & r_{h}
\end{array}\right) t\right) \\
u_{x}^{\prime}(r,, x, t)=\frac{m_{g}}{r k_{x}} u^{\prime}(r,, x, t)
\end{array}\right.
$$

In Eq. (2), $A$ is the gust amplitude, $m_{g}$ is the gust azimuthal order, and the radial wave number is given by $k_{r}=\frac{2 q}{r_{t} \quad r_{h}}$, where $q$ is an integer, $r_{h}$ and $r_{t}$ are the inner and outer radius, respectively.

In the presence of spinning gusts (discarded in Eq. (1)), a suited axial component of disturbance velocity, $u_{x}^{\prime}$, has to be also injected to ensure the divergence-free condition.

The following parameters are considered:

$$
r_{h}=24 / 4 \pi(\mathrm{m}), r_{t}=2 r_{h}(\mathrm{~m}), f_{0}=\omega / 2 \pi=177.5(\mathrm{~Hz}), A=0.1, U_{0}(\mathrm{~m} / \mathrm{s}), \text { and } m_{g}=16
$$

Applying the well-known Tyler \& Sofrin $\operatorname{condition}^{33}\left(m=n B \quad k V\right.$, with $\left.B=m_{g}\right)$, a largely dominant acoustic mode with azimuthal order $m=-8$ is expected. Thus, the CAA domain can be restricted to a $2 \pi / 8$ angular sector covering 3 vane channels and periodicity conditions can be applied in the azimuthal direction. A 3D view of the mesh is shown in Fig. 1. The grid is extending from -4 chords (upstream) to 12 chords in the axial direction, and a very fine grid spacing of about 1/500 chord is imposed in the vicinity of the leading and trailing edges. Respectively 370, 46, and 181 cells are used in the axial, radial, 
and azimuthal directions, which totalizes 3.2 M points. Since Tam's BC are not actually able to fully avoid reflections of outgoing spinning acoustic modes, a local stretching of the cells (with a coefficient equal to 1.03) associated to a sponge zone is applied at the exit (downstream) of the domain. A converged solution requires about 30 hours over 120 processors.

Typical snapshots of the computed disturbance fields (tangential velocity and pressure) for the cases $q=0$ and $q=3$, repeated over a full revolution, are presented in Fig. 2. The expected dominant acoustic cut-on mode $m=-8$ is clearly identified, and the damping zone mentioned above allows making the sound waves exit the domain without creating noticeable numerical reflections.

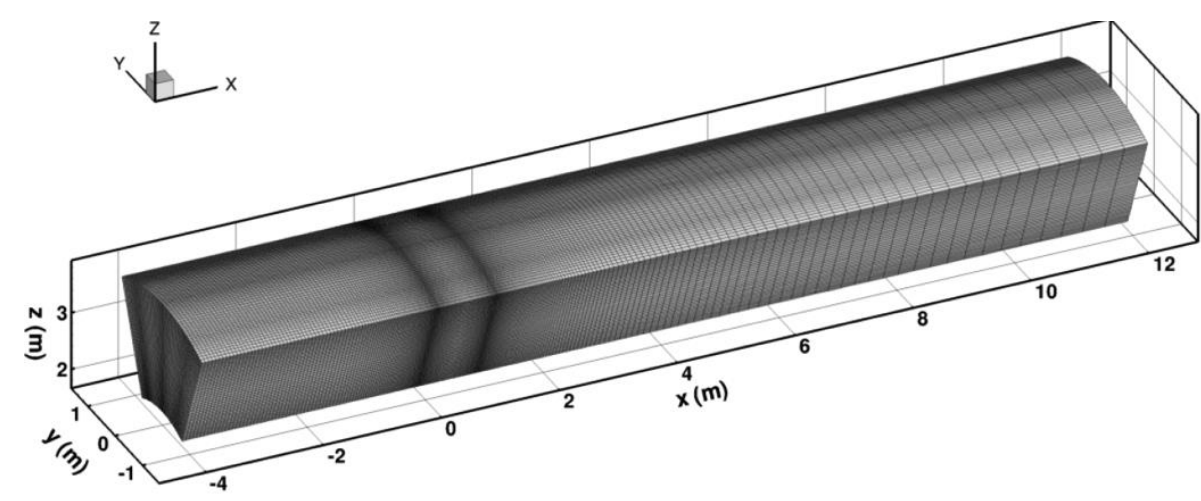

Figure 1. 3D annular grid (3 vane channels) used for the CAA benchmark

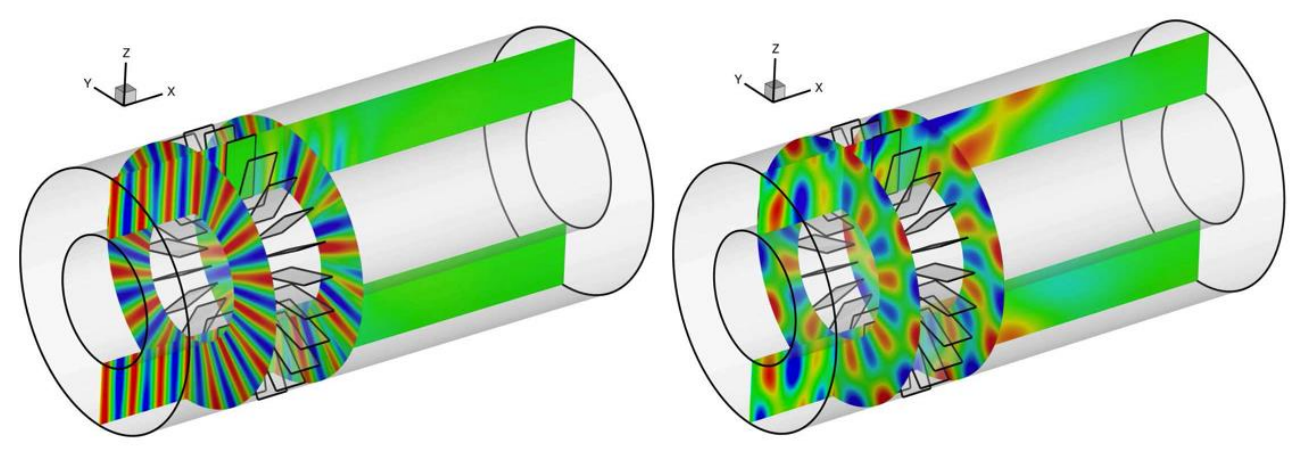

a) 

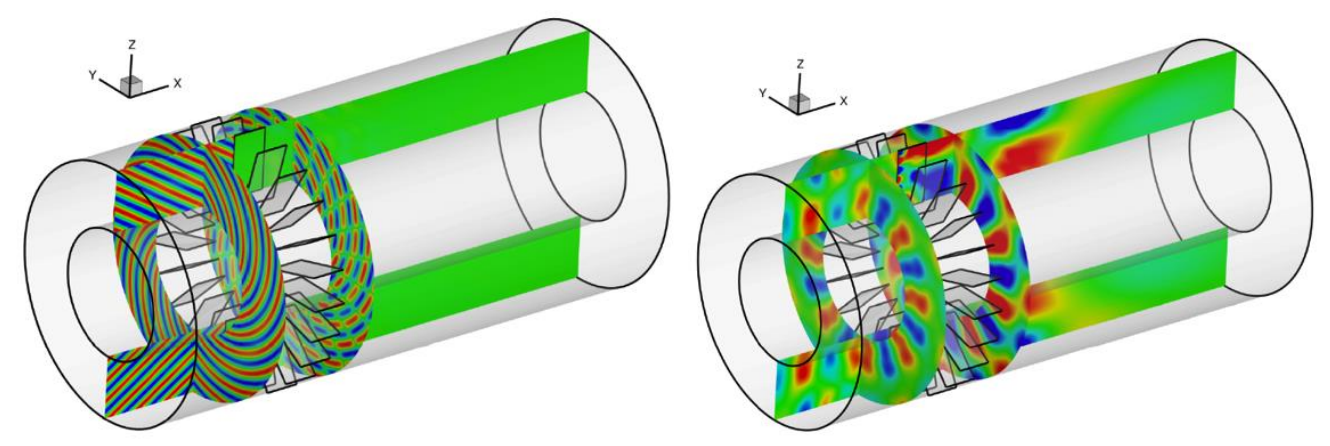

b)

Figure 2. Snapshots of tangential velocity disturbances $( \pm 17 \mathrm{~m} / \mathrm{s}$, left $)$ and pressure disturbances ( $\pm 2000 \mathrm{~Pa}$ (a) \& $\pm 300 \mathrm{~Pa}$ (b), right) duplicated over a full revolution for cases $q=0$ (a) and $q=3$ (b)

Radial distributions over the vane surface of the harmonic wall pressure component $\left(f=f_{0}\right)$ provided by CAA are compared to available semi-analytical solutions of Schulten ${ }^{17}$ in Fig. 3, for case $q=3$. The agreement is excellent, with only slight differences close to the trailing edge $(x=0.9 c)$.

Finally, the modal amplitude and phase of the acoustic pressure obtained from a Fourier-Bessel transformation over a selected cross-section at $x=2 c$ ( 2 chords downstream the cascade), are compared in Figure 4 to the solutions of Namba \& Schulten ${ }^{15}$ for $q=0,1,2,3$ and cut-on modes $(-8,1),(-8,2)$, $(-8,3)$. Again, a fairly good agreement is observed for all cases. 


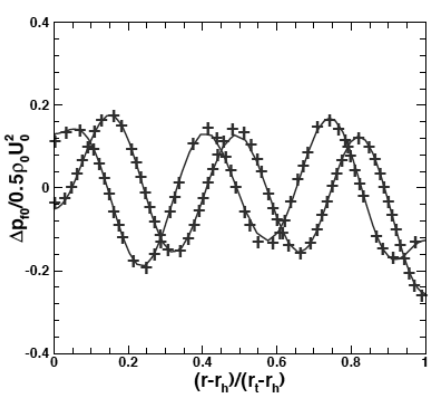

(a) $x=0.06 c$

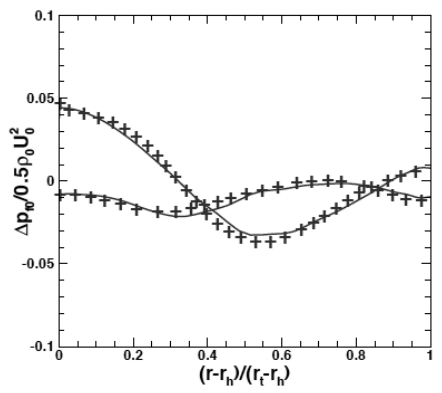

(c) $x=0.5 c$

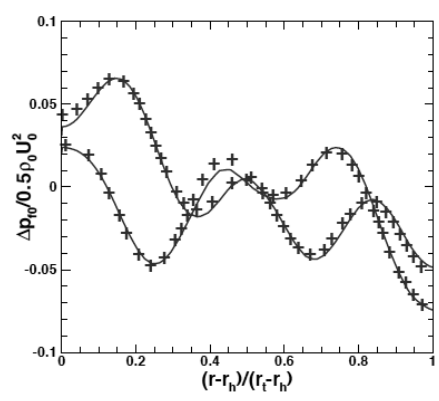

(b) $x=0.2 c$

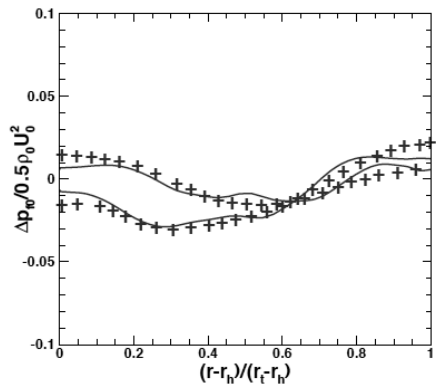

(d) $x=0.9 c$

Figure 3. Harmonic normalized pressure over the vane surface for $q=3$ :

CAA results (-) compared to Schulten results (+) ; Real part (light) and imaginary part (dark)
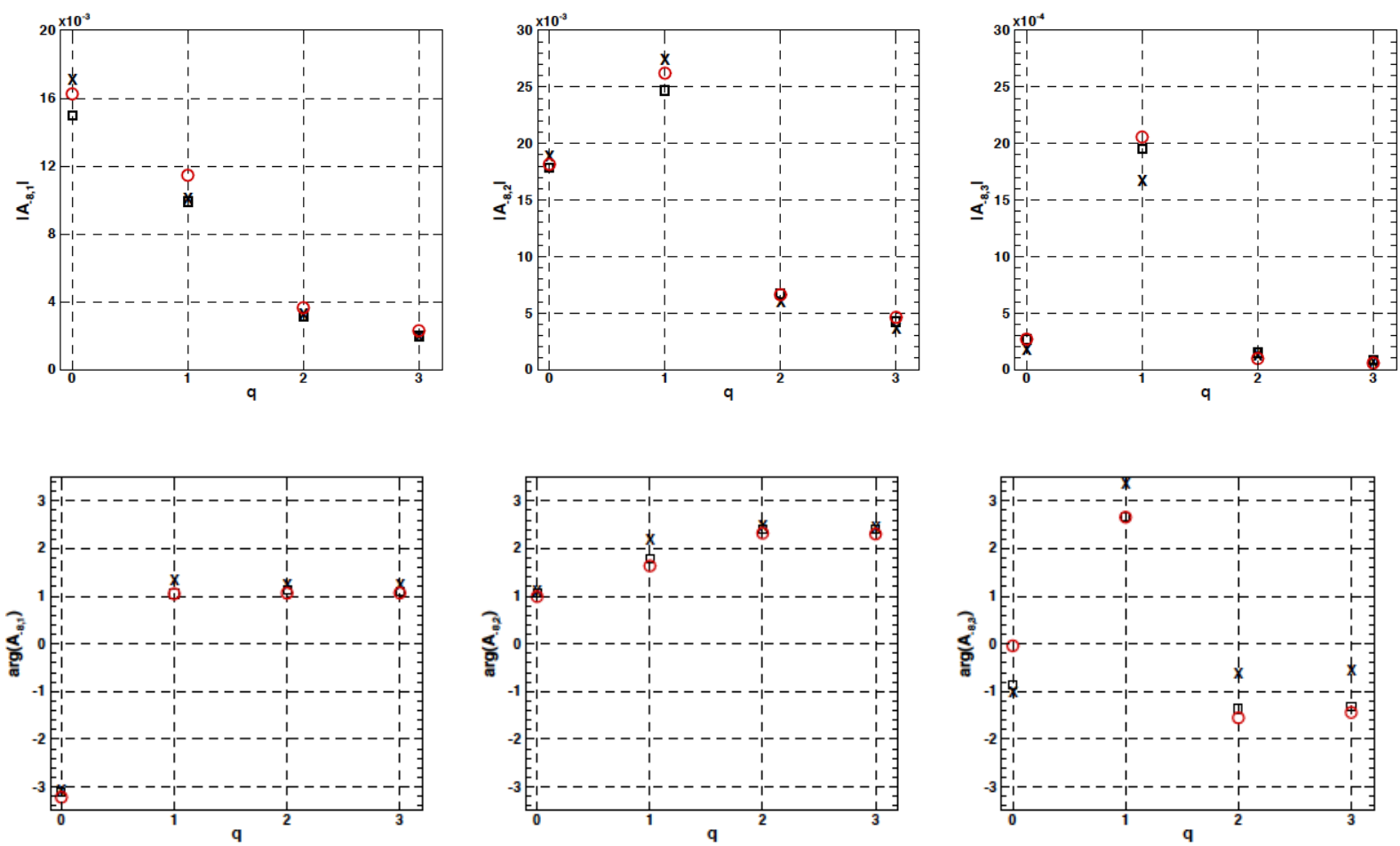

Figure 4. Modal amplitude (top) and phase (bottom) issued from Fourier-Bessel transform at $x=2 c$ : $m=-8(n=1$ to 3$)$ and $q=0,1,2,3$; CAA results (O) compared to Schulten ( $\square$ ) and Namba (X) 


\section{Applications to turbulence-annular cascade configurations}

The previous single harmonic gust simulations have been extended to broadband noise by considering a synthetic turbulent inflow obtained from an HIT spectrum. Two application cases are discussed below, considering purely axial and swirling mean flows, respectively.

\section{A. Turbulence-annular cascade interaction in a uniform axial mean flow}

\section{A.1 Experiment and analytical solution}

A first validation case is devoted to a turbulence-cascade interaction using a turbulence grid in a purely axial mean flow, related to an experiment proposed by ECL ${ }^{18}$. A picture of the anechoic open-jet wind tunnel with an outlet view of the model and a sketch of the test rig are shown in Fig. 5.
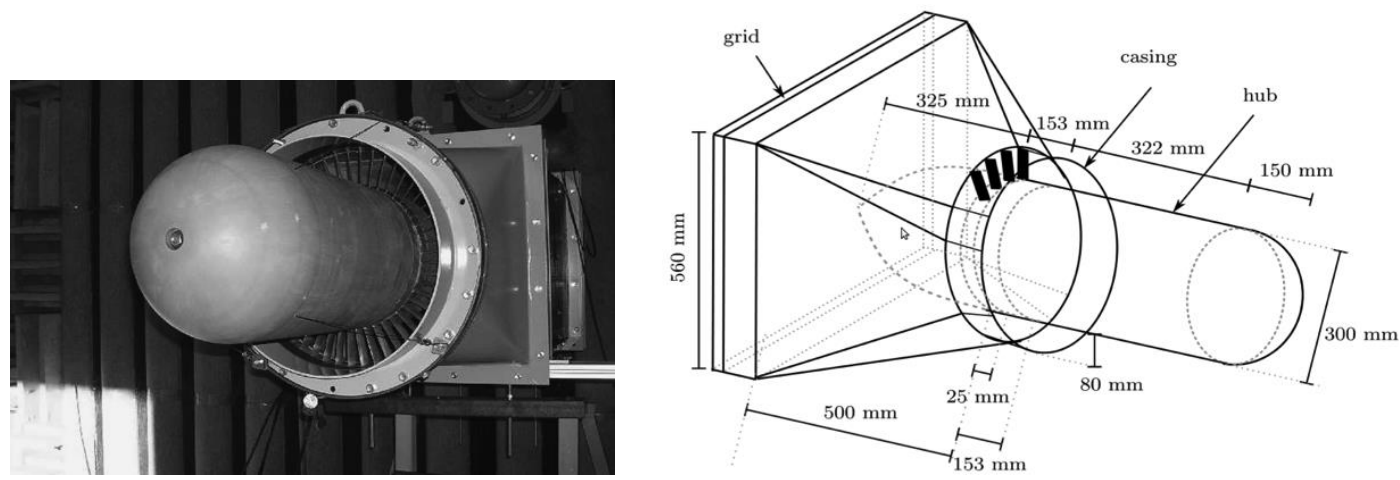

Figure 5. ECL open-jet anechoic wind tunnel experiment (left) and sketch of the rig (right)

Two selected turbulence grids $(\mathrm{T} 1, \mathrm{~T} 2)$ with respective averaged turbulence intensity $T_{u} \approx 3.5 \%$ and $T_{u} \approx 6 \%$, and two cascades $(\mathrm{C} 1, \mathrm{C} 2)$ with respective vane numbers $V=49$ and $V=98$ were investigated. The flat plate vanes have an $L=80 \mathrm{~mm}$ span, a $c=25 \mathrm{~mm}$ chord and a $=16.7^{\circ}$ stagger angle (with a zero degree angle of attack). The inner and outer radii of the annular duct are respectively $r_{h}=150 \mathrm{~mm}$ and $r_{t}=230 \mathrm{~mm}$, and the mean (axial) velocity is $U_{0}=80 \mathrm{~m} / \mathrm{s}$. T1 and T2 grids gave an estimated integral length scale $\Lambda$ around $20 \mathrm{~mm}$ when fitting the hotwire measurements to the Liepmann HIT model.

The 2-wave number Liepmann spectrum expressed in cylindrical coordinates in the $x$-duct frame writes: 


$$
u \text { u }\left(k_{x}, k_{r}\right)=\frac{3 \bar{u}^{2}{ }^{2}}{4} \frac{k_{x}^{2}{ }^{2}+k_{r}^{2}{ }^{2}}{\left(1+k_{x}^{2}{ }^{2}+k_{r}^{2}{ }^{2}\right)^{5 / 2}}
$$

In Eq. (3), the turbulent upwash velocity, $u$, is related to the turbulence intensity, $T_{u}$, as $\bar{u}^{2}=T_{u}^{2} U_{0}^{2}$. CAA simulations have been focused on $\mathrm{T} 2-\mathrm{C} 1$ and $\mathrm{T} 2-\mathrm{C} 2$ cases, for which the main parameters are summarized in Tables $1 \mathrm{a}$ and $1 \mathrm{~b}$. It can be noticed that turbulence flow characteristics generated by the turbulence grid are slightly modified when changing the cascade vane number (present values for cascade C2 used here are expected to be more representative than those reported in Ref. [18], in which same values were considered for grids $\mathrm{C} 1$ and $\mathrm{C} 2$ ).

Table 1a. Annular cascade geometry and flow considered in the CAA

\begin{tabular}{|c|c|c|c|c|c|}
\hline$r_{h}(\mathrm{~mm})$ & $r_{t}(\mathrm{~mm})$ & $c(\mathrm{~mm})$ & $L(\mathrm{~mm})$ & $\left(^{\circ}\right)$ & $U_{0}(\mathrm{~m} / \mathrm{s})$ \\
\hline 150 & 230 & 25 & 80 & 0 & 80 \\
\hline
\end{tabular}

Table 1b. Radially-averaged turbulence data used in the CAA

\begin{tabular}{|c|c|c|c|c|}
\hline Grid-Cascade & $\begin{array}{c}\mathrm{T} 1-\mathrm{C} 1 \\
(\mathrm{~V}=49)\end{array}$ & $\begin{array}{c}\mathrm{T} 1-\mathrm{C} 2 \\
(\mathrm{~V}=98)\end{array}$ & $\begin{array}{c}\text { T2-C1 } \\
(\mathbf{V}=\mathbf{4 9})\end{array}$ & $\begin{array}{c}\text { T2-C2 } \\
(\mathbf{V}=98)\end{array}$ \\
\hline $\mathrm{T}_{\mathrm{u}}(\%)$ & 3.6 & 3.4 & $\mathbf{6}$ & $\mathbf{5 . 5}$ \\
\hline$\Lambda(\mathrm{mm})$ & 17.6 & 22 & $\mathbf{2 0}$ & $\mathbf{2 5 . 6}$ \\
\hline
\end{tabular}

For the sake of simplicity, the stagger angle is set to zero in the simulations because its effect on turbulence-airfoil noise, for small values, is known to be negligible. Indeed, calculations of sound power spectra in the outlet duct issued from the Amiet-based code developed by Reboul ${ }^{23,24}$ (considering an isolated airfoil response model and a Green's function valid for annular ducts) and setting $=0^{\circ}$ or $=16.7^{\circ}$ provide almost identical results (see Fig. 6). This Amiet-based formulation is detailed in the Appendix. Furthermore, the incoming turbulence can be restricted to parallel gusts $\left(k_{r}=0\right.$ in Eqs. (1) and (3)), as done in Amiet's theory and suggested in Ref. [24] for turbofans with span-to-chord ratio $L / c>3$. 
This assumption was also verified numerically by Clair $^{8}$ for turbulence-airfoil simulations. As explained in Ref. [8], this is simply achieved in the CAA by setting $\quad k_{r}=\frac{2}{L}$ in Eq. (1).

The Amiet-based results are compared to solutions obtained by Posson ${ }^{18}$ and Zhang ${ }^{25}$ in Fig. 7, and to experiment too. The T1-C1 case was recently investigated by Zhang ${ }^{25}$ who addressed a quite relevant solution based on the lifting surface method of Schulten ${ }^{17}$, generalized to broadband noise. Zhang's result for the $\mathrm{T} 1-\mathrm{C} 1$ case has been extrapolated to the $\mathrm{T} 2-\mathrm{C} 1$ case in Fig. $7 \mathrm{~b}$ by simply applying a frequency depending correction factor that is equal to the ratio of corresponding Liepmann spectra. For both cases, the three predictions are reasonably close with a 3-4 dB over-estimate of Reboul's results compared to those of Zhang, that can be partly attributed to cascade effects too neglected in Amiet's isolated airfoil theory, although Amiet-based predictions better fit the experiment (the low-frequency hump beyond $500 \mathrm{~Hz}$ visible on the experimental spectra has to be related to an additional noise caused by installation effects $^{18}$ ). The 3D lifting surface method expected to be the most rigorous one, provides rather similar results to the quasi-3D cascade model of Posson in the high frequency range, whereas the PWL spectrum of Reboul displays a lower level attenuation slope.

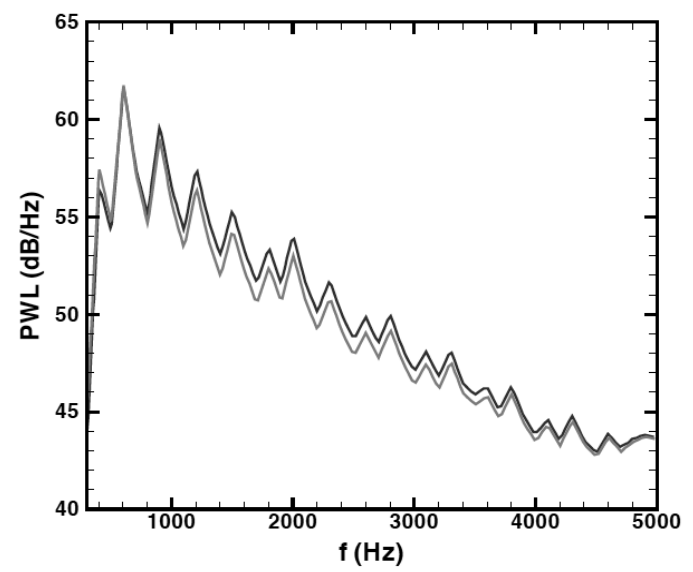

Figure 6. PWL spectra $(\mathrm{dB} / \mathrm{Hz})$ in the outlet duct provided by Amiet-based calculation on T2-C 1 case and setting $\quad=16.7^{\circ}$ (light) or $\quad=0^{\circ}$ (dark) 

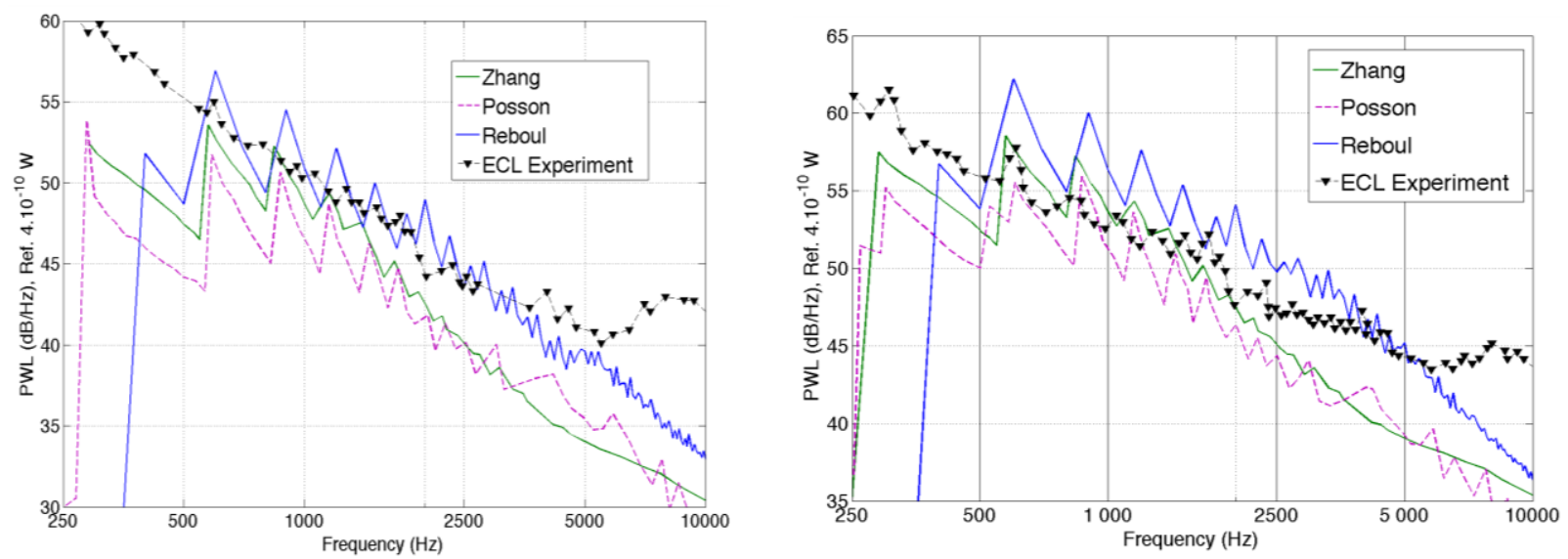

Figure 7. Comparison of outlet PWL spectra obtained by three different calculation methods applied to ECL experiment (raw measurements) for T1-C1 (left) and T2-C1 (right) cases

\section{A.2 Mesh generation}

The use of a simplified turbulence spectrum representation, $\left(k_{x}, 0\right)$, without azimuthal dependence, allows us to limit the CAA domain to a single vane channel by applying suitable periodicity conditions in the angular direction (and so to greatly reduce the CPU costs). Thus, the CAA domain is restricted to a $2 \pi / V$ sector leading to a $3 \mathrm{D}$ grid of about $1.5 \mathrm{M}$ cells. The CAA grid characteristics are summarized in Table 2. The mesh for cascade $\mathrm{C} 2(\mathrm{~V}=98)$ is simply achieved by reducing by half the angular spacing used for $\mathrm{C} 1$ so that the number of grid points in each direction remains the same. 3D and section views of the CAA grids are shown in Fig. 8. The synthetic turbulent inflow is injected for frequencies ranging from $300 \mathrm{~Hz}$ to $5000 \mathrm{~Hz}$, with a frequency resolution $\Delta f=100 \mathrm{~Hz}$. Hence, a complete period $T=1 / \Delta f$ is achieved after 85,000 time iterations, requiring about 27 hours over 64 processors for the two cases, and converged statistics are obtained after only 2 periods.

Table 2. CAA grid characteristics

\begin{tabular}{|c|c|c|c|c|c|}
\hline$N_{x}$ & $N_{r}$ & $N_{\theta}$ & Axial extent $(m)$ & radial extent $(m)$ & $\begin{array}{c}\text { Azimuthal extent } \\
\text { (radians })\end{array}$ \\
\hline 373 & 51 & 81 & $-0.1<x<0.25$ & $0.15<r<0.23$ & $-\pi / 49<\theta<\pi / 49$ \\
$-\pi / 98<\theta<\pi / 98$
\end{tabular}



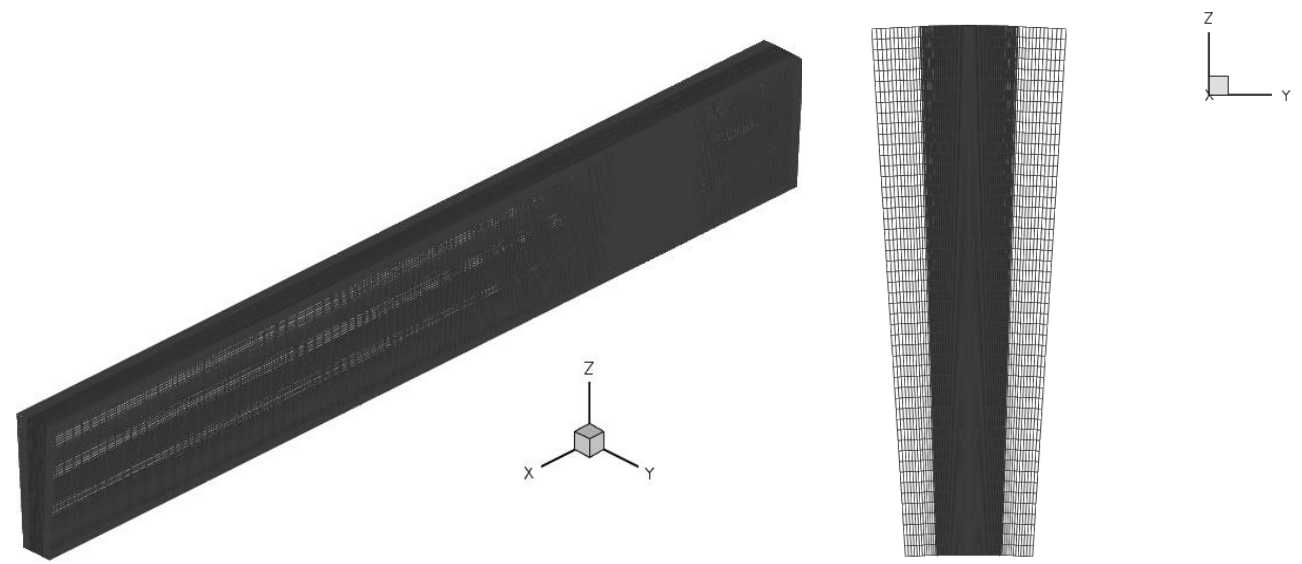

Figure 8. 3D view (left) and section view (right) of CAA grids for cascade C1 (light) and C2 (dark)

\section{A.3 T2-C1 simulation}

Although Amiet's theory is only valid for an isolated airfoil, cascade effects should be taken into account here by the use of periodicity conditions, traducing the influence of adjacent vanes on the aerodynamic response of the airfoil. However, the restriction to planar gusts (which is similar to setting $m_{g}=0$ in Eq. (2)), does not allow anymore to assess the acoustic response directly, as done with harmonic gusts in section III. Indeed, only interaction modes $m= \pm k V$ can be created, and as turbulence-airfoil interactions occur in-phase for all vanes, interference effects between dipole-sources of adjacent vanes lead to a quasi null radiated field. This point is illustrated in Fig. 9, showing snapshots of azimuthal velocity disturbances (Fig. 9 left) and pressure disturbances (Fig. 9 right) over a 3D annular slice corresponding to the CAA domain duplicated over 3 angular sectors. The planar shape of the multiharmonic gusts is clearly highlighted as well as the dipolar source response of each vane, giving rise to a noise cancellation in the upstream and downstream directions due to destructive interference effects. Anyway, the fluctuating wall pressure over the vane surface is expected to be reliable, and the radiated sound field can be computed by means of a FWH analogy. This is practically achieved by chaining the CAA output to an in-house code solving the loading noise term of FWH formulation (with an in-duct modal Green's function) written in the frequency domain.

The RMS surface pressure over the vane is plotted in Fig. 10 showing an expected source concentration in the leading edge region. Chordwise RMS pressure profiles (normalized by ${ }_{0} U_{0}^{2}$ ) 
computed by the CAA at hub (light), mid-span (medium) and casing (dark) locations, are compared to the Amiet-based (isolated airfoil) solution in Fig. 11. A reasonably good agreement can be observed, but the levels of the computed profiles are slightly below the Amiet-based solution: this might be attributed to cascade effects that are taken into account in the CAA.

Finally, in Fig. 12 the PWL spectrum provided by CAA-FWH calculation is compared to Zhang's semi-analytical solution, and to the measurements for which corrected data (partial filtering of extra sources contribution suggested in Ref. [17]) are also addressed. The numerical prediction is very close to Zhang's solution, both spectra revealing an accurate capture of the peaks even if the levels are a little bit higher compared to the corrected experiment. Furthermore, the numerical prediction displays a slightly lower level attenuation slope that better fits the experiment beyond $2000 \mathrm{~Hz}$ comparing to the lifting surface method. The PWL reduction of about $3 \mathrm{~dB}$ compared to the Amiet-based solution of Reboul (Fig. 8, right), is in accordance with the wall pressure analyzes discussed in Fig. 11.
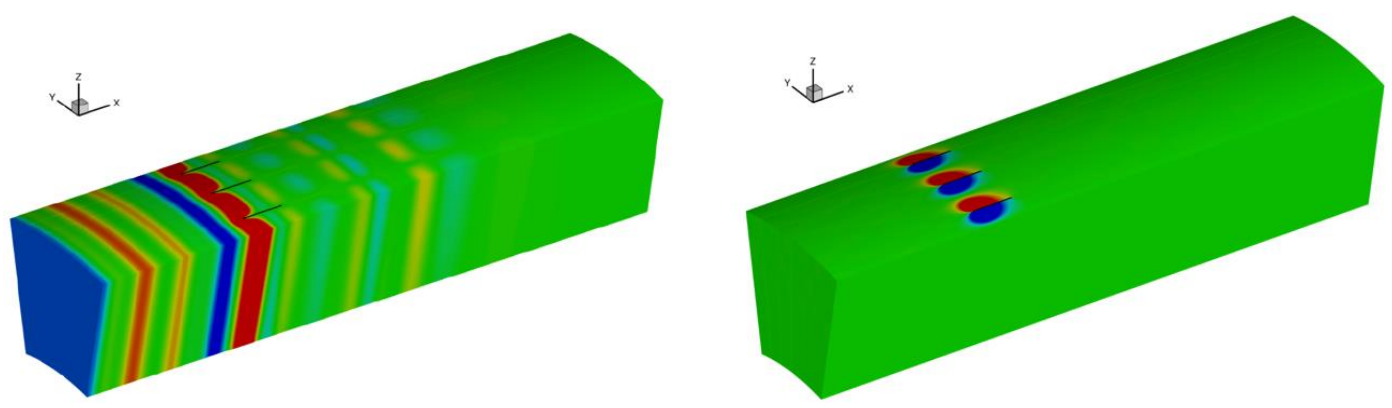

Figure 9. Snapshot of azimuthal velocity disturbances $( \pm 2.5 \mathrm{~m} / \mathrm{s}$, left $)$ and pressure disturbances $( \pm 100 \mathrm{~Pa}$, right) duplicated over 3 angular sectors for the case T2-C1

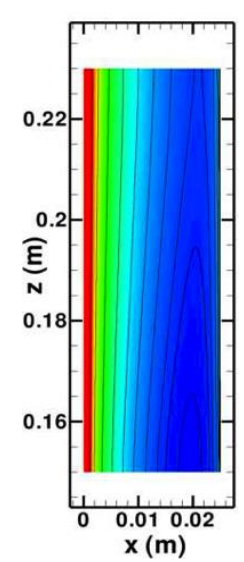

Figure 10. RMS vane surface pressure (0-300 Pa)

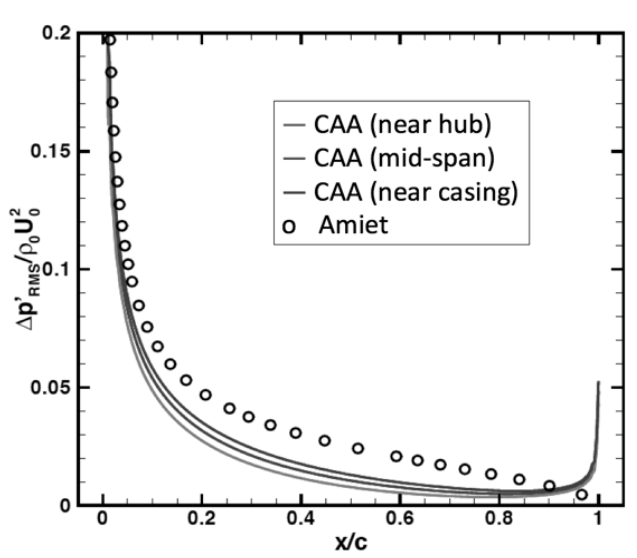

Figure 11. Chordwise RMS pressure profiles (normalized) issued from CAA and Amiet 16

American Institute of Aeronautics and Astronautics 


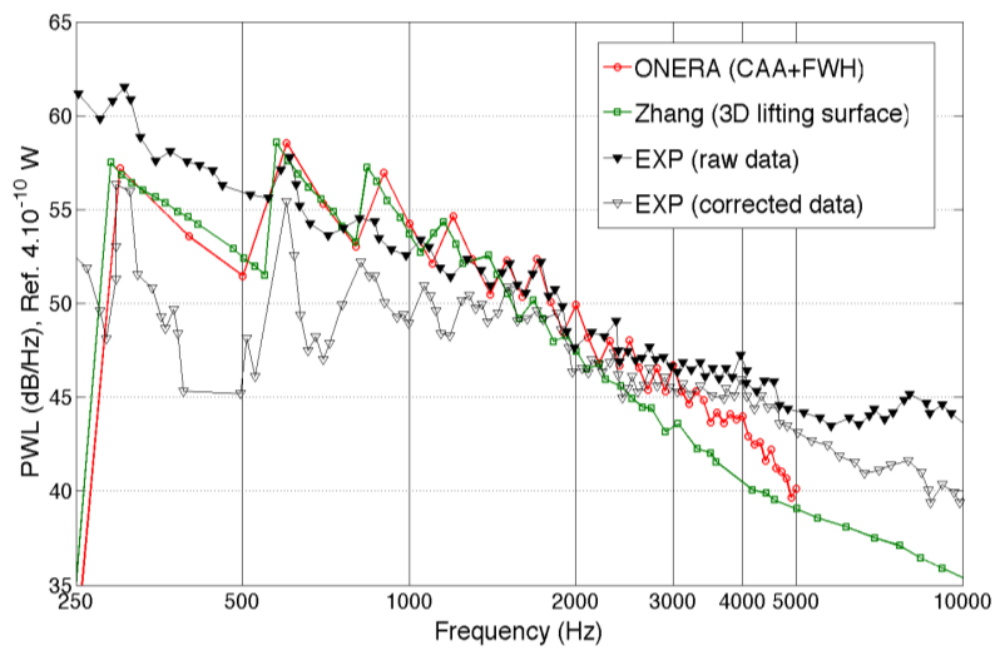

Figure 12. Outlet duct PWL spectra provided CAA+FWH, semi-analytical method (Zhang) and compared to experiments (raw and corrected measurements) for T2-C1 case

\section{A.4 T2-C2 simulation}

A second simulation has been performed on T2-C2 case with similar analyzes. A comparison of the pressure profile along the vane chord at mid-span in presented in Fig. 13(a), revealing a much lower amplitude of the cascade $\mathrm{C} 2$ response compared to $\mathrm{C} 1$. The estimated PWL spectrum obtained by the coupling with FWH integral (and applying a non-coherent sum over 98 vanes instead of 49) is compared to the previous result in Fig. 13(b). Surprisingly, the cascade C2 is found to be less noisy than C1, suggesting a very strong cascade effect. Regarding to the cascade C2 geometry, the inter-vane distance at mid-span is equal to $12 \mathrm{~mm}$, which is about half the size of the turbulence integral length scale. For this reason, unusual intense acoustic coupling between vanes is suspected including correlation effects between adjacent vanes (whereas vanes are assumed to be fully uncorrelated in the FWH formulation). It is also important to notice that although turbulence intensity is almost the same, the mean value of the turbulence integral length scale for T2-C2 case $(\Lambda=25.6 \mathrm{~mm})$ is $25 \%$ higher than $\mathrm{T} 2-\mathrm{C} 1$ case $(\Lambda=20 \mathrm{~mm})$ giving rise to a significant modification of the cascade response (independently to the vane number ratio). Thus, PWL amplification from cascade $\mathrm{C} 1$ to $\mathrm{C} 2$ provided by Amiet-based predictions (neglecting cascade effects) plotted in Fig. 14 do not highlight the expected $10 \log (\mathrm{V})$ law with isoturbulence (dashed line), under the present experimental conditions. 
The PWL spectrum numerically obtained is then compared to Zhang's solution and to the experiment in Fig. 15. Both predictions are found to be in a rather good agreement, although the PWL issued from lifting surface method is 3-4 dB higher in the low frequency range [300-1000 Hz]. The two solutions become almost identical beyond $1.5 \mathrm{kHz}$ whereas they are drifting away from the measurements that are displaying a much lower attenuation slope. On the other hand, Onera prediction seems to better fit the measurements (more particularly the corrected data) up to $1.5 \mathrm{kHz}$.

Finally, PWL amplifications/attenuations (adopting a linear scale for frequency) provided by calculations are compared to those deduced from the measurements in Fig. 16. An important dispersion of the results with significant level differences can be observed, and no method is actually able to match the experimental results (Amiet-based solution being the closer one to corrected data, which is not realistic and makes these corrections not fully reliable). However, the three predicted spectra exhibit similar shapes, with a strong increase at lower frequencies and then tending to an asymptotic behaviour at high frequency. Zhang's predictions display a quasi-null PWL amplification from $1 \mathrm{kHz}$ to $3 \mathrm{kHz}$ and then an attenuation (reaching $-2 \mathrm{~dB}$ ), which tend to confirm the trend of our numerical results, even if we predict a higher attenuation with a level shift within 1.5 to $3.5 \mathrm{~dB}$.

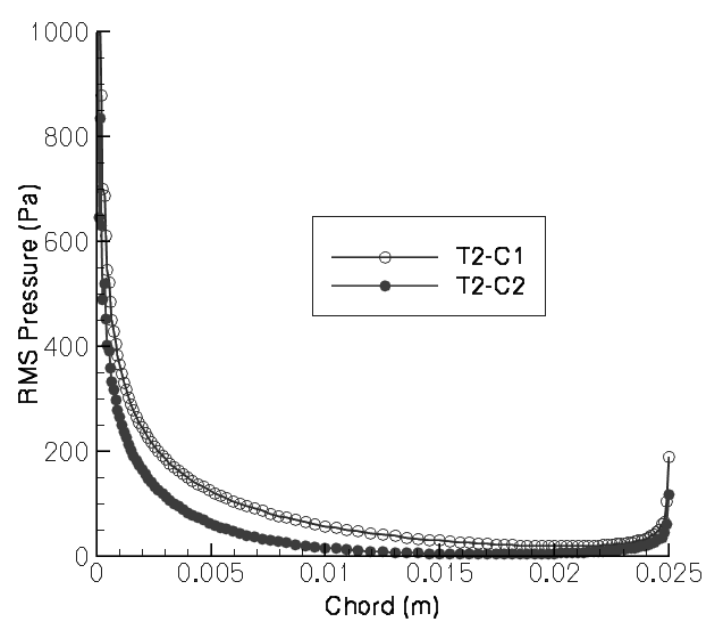

a) RMS wall pressure along chord (at mid-span)

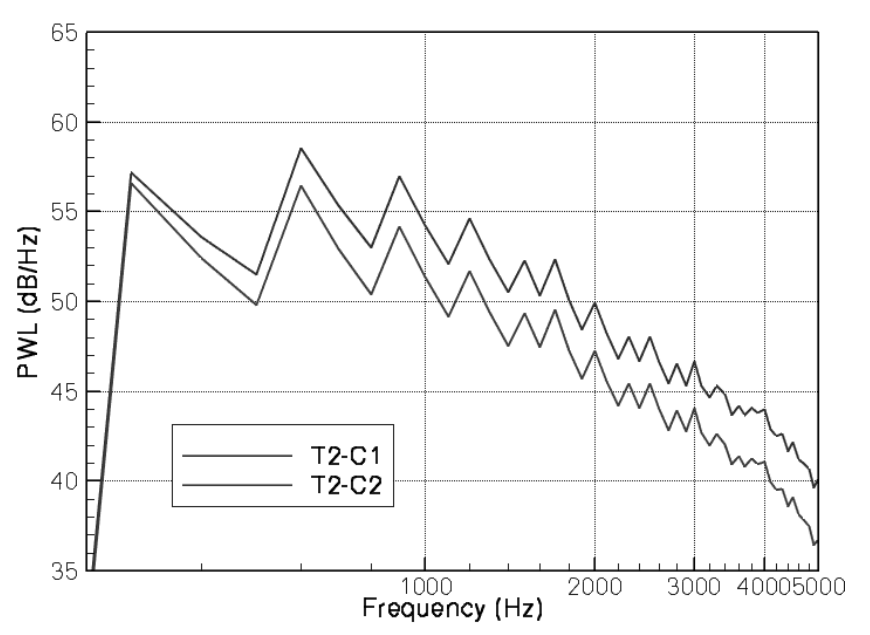

b) PWL spectra in the outlet duct

Figure 13. Comparaison of numerical simulation results between $\mathrm{T} 2-\mathrm{C} 1$ (light) and T2-C2 (dark) cases 


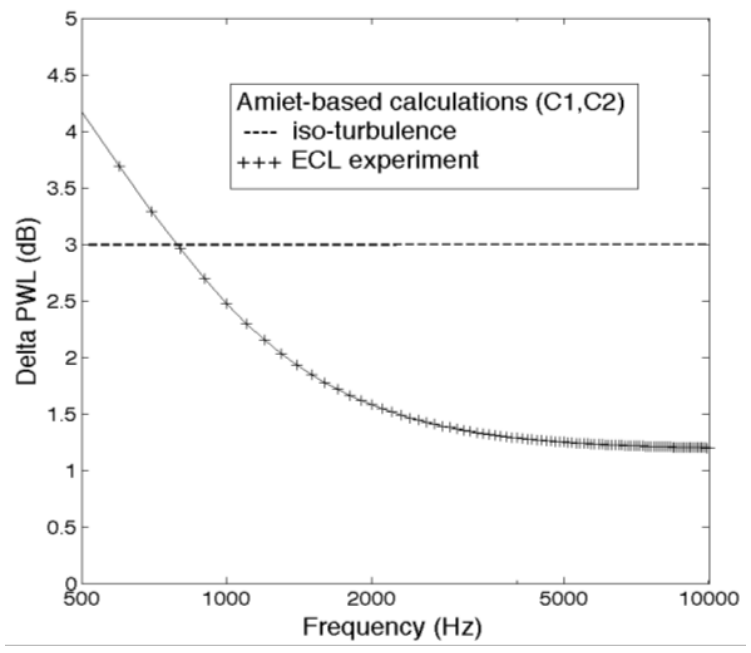

Figure 14. PWL amplification (dB) from cascade $C 1$ to $C 2$ obtained from Amiet-based ONERA code considering iso-turbulence or ECL experiment conditions (T2 grid)

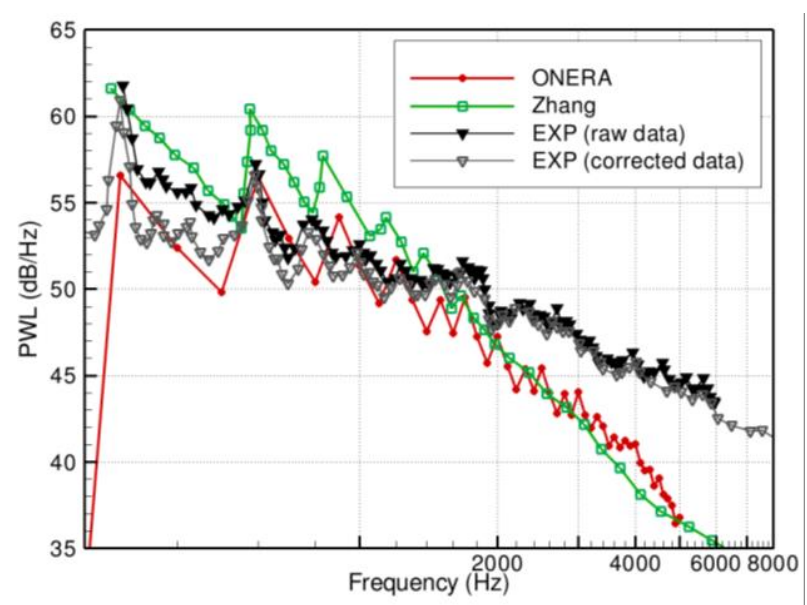

Figure 15. Outlet duct PWL spectra provided CAA+FWH (ONERA), semi-analytical method (Zhang) and compared to experiments (raw and corrected measurements) for T2-C2 case 


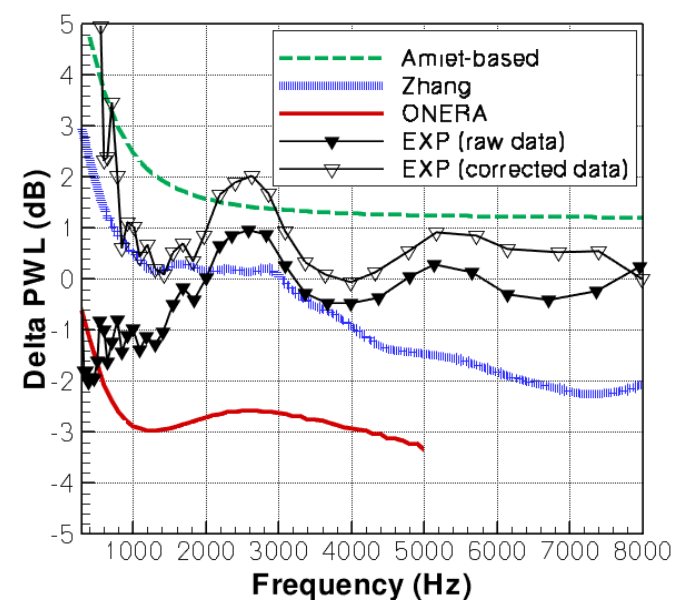

Figure 16. PWL amplification or attenuation (in $\mathrm{dB}$ ) issued from calculations and measurements related to $\mathrm{T} 2-\mathrm{C} 1$ and $\mathrm{T} 2-\mathrm{C} 2$ cases

\section{A.5 Complete turbulence spectrum $\left(k_{r} \neq 0\right)$}

In order to check our numerical predictions, the previous simulations have been run again by considering the complete turbulence spectrum given by Eq. (1), including the radial wave numbers $\left(k_{r}\right)$. For that purpose, as suggested in conclusions of Ref. [11], the code $s A b r i n A . v O$ has been modified to speed up the turbulent-like flow generation process by externalizing part of the source terms (read from an input data file) involved in Tam's inflow boundary condition ${ }^{8,14}$. By this way, the CPU time involved for the generation of the turbulence is negligible compared to the CAA (only source-term derivatives of Tam's BC are numerically calculated in the code), so that the total CPU time for the complete spectrum $\left(k_{r} \neq 0\right)$ is comparable.

The radial wave number spacing $\Delta k_{r}$ is set equal to $2 \pi / L(L=80 \mathrm{~mm})$ and the maximum number of radial modes ( $2 M$ in Eq. (1)) is related to the CAA grid density (in the spanwise direction) at the inflow boundary. Here, 50 regular radial planes are used so that 26 radial modes ( $2 \mathrm{M}$ modes plus parallel gust) are imposed, referring to Shannon criterium. Here, as $U_{r}=0$ and $u_{r}=p=0$ for the synthetic turbulent field, there are no terms involving radial derivatives in the LEE and thus the 10-points-per-wavelength criterion is not limiting in this direction during the convection of the incoming perturbations. However, one should note that the direct acoustic radiation from the vanes (not solved with the present method) 
might not be accurately captured by the CAA for the higher radial modes. As for previous computations, convergence is achieved after 2 complete periods and the total required CPU time (54 hours over 64 processors) is almost the same. Typical 3D map snapshots of azimuthal velocity and pressure disturbances are shown in Fig. 17. In comparison to Fig. 9 (left), the synthetic turbulent flow (Fig. 17, left) provided by the present stochastic model highlights random patterns in both axial and radial directions. Here again, the pressure disturbance field reveals a dipole source concentrated at the leading edge of the vane and a quasi null radiated sound field (for the same reason discussed before). It can be noticed that the pressure levels are much lower than for the T2-C1 case. Moreover, some spurious spots can be observed near the bottom corner of the CAA domain exit probably due to remaining numerical reflections. In Fig. 18 (a), RMS vane pressure map predicted using the present synthetic turbulence (including oblique gusts) for the T2-C2 case is compared to the one obtained using parallel gusts (Fig. 18 (a), left) showing very similar patterns and levels despite a slightly more oscillating solution in the spanwise direction (Fig. 18 (a), right). The pressure PSD near the leading edge of the vane for two radial positions (10\% and 50\% of the span) are compared in Fig. 18 (b). The solutions obtained with the complete 2D turbulence spectrum (solid lines) display high oscillations compared to the ones issued from turbulent inflow restricted to axial modes (dashed lines). This is due to interference effects between radial modes and the fact that no averaging (that would require several runs with different sets of random phases) has been realized. Note that statistical errors could be simply reduced by integrating the PSD levels over consecutive spectral bands as done in Ref. [8]. Anyway, the fluctuating levels of the spectra seem to oscillate around a mean value that roughly fits the smooth solution, although higher levels at high frequencies can be observed. 


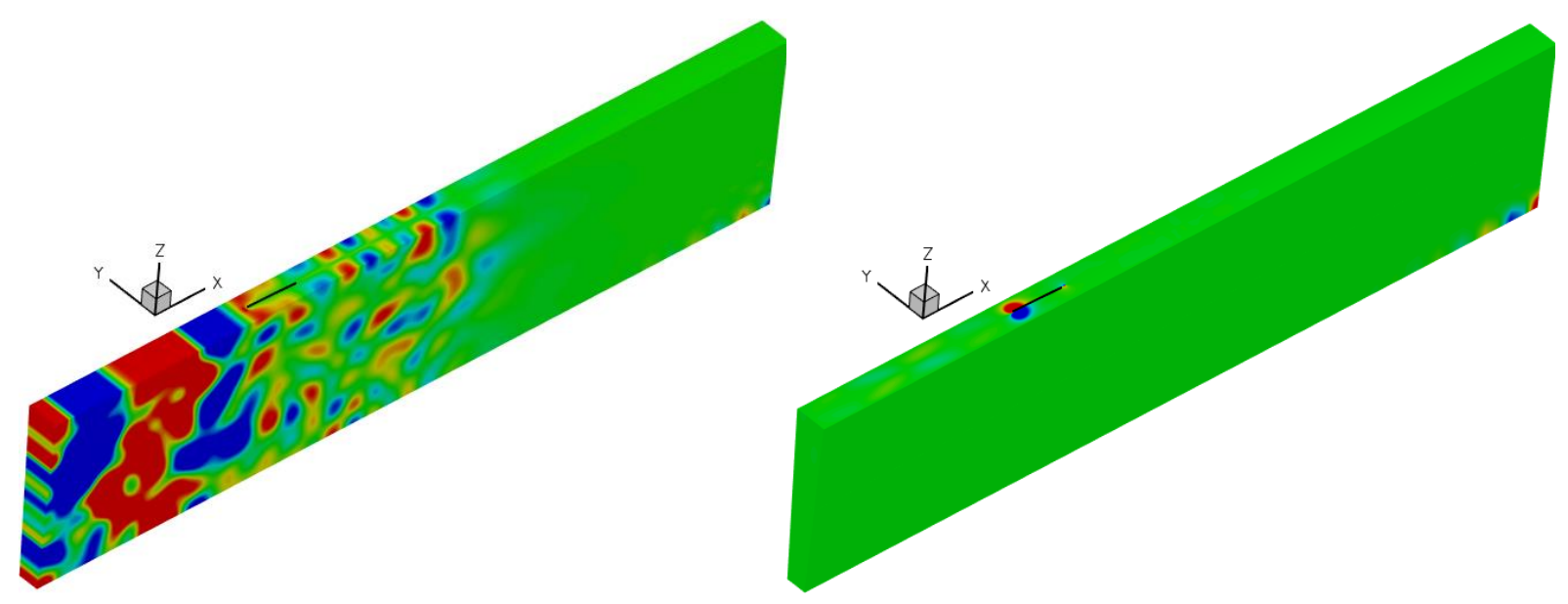

Figure 17. Snapshot of azimuthal velocity disturbances $( \pm 2.5 \mathrm{~m} / \mathrm{s}$, left $)$ and pressure disturbances ( $\pm 25 \mathrm{~Pa}$, right) for the case T2-C2 using synthetic turbulence with radial wave numbers

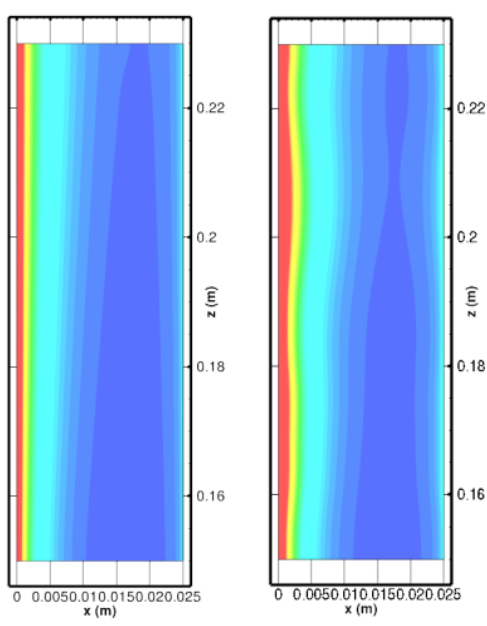

a) RMS vane surface pressure (0-300 $\mathrm{Pa})$

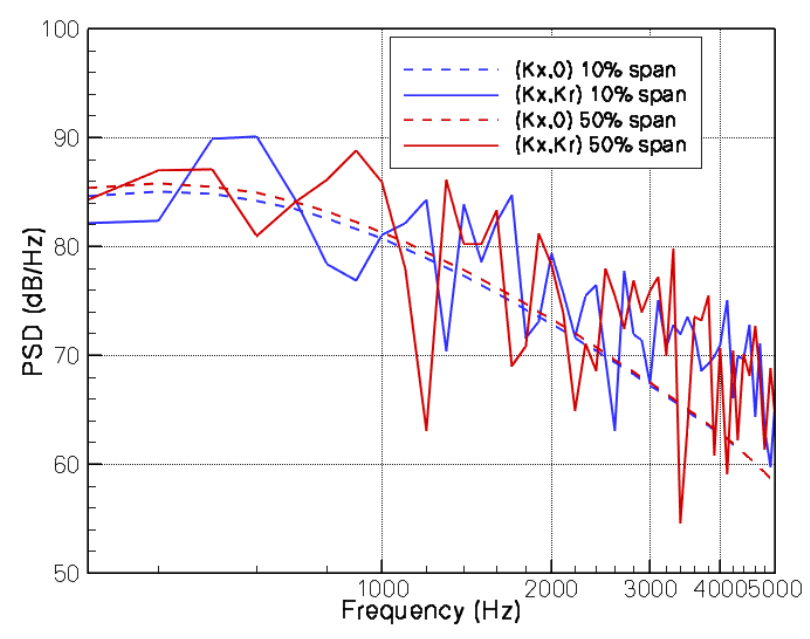

b) Pressure PSD $(\mathrm{dB} / \mathrm{Hz})$ near the leading edge

Figure 18. Comparison of CAA predictions using synthetic turbulence without ((a) left, (b) dashed lines) and with ((a) right, (b) solid lines) radial wave numbers for T2-C2 case

Finally, The PWL spectra in the outlet duct predicted by the coupling with a FWH integral for both approaches are compared in Fig. 19 for the two cases T2-C1 (light) and T2-C2 (red). Despite the oscillations present in the pressure PSD, the radiated PWL spectra using $\left(k_{x}, k_{r}\right)$ turbulence spectrum (plotted in solid lines) are almost identical to the previous ones (in dotted lines), excepted a slight increase of the levels beyond $2 \mathrm{kHz}$, consequently to the PSD levels increase observed in Fig. 18 (b). Thus, the cascade effects highlighted in Fig. 16 are not expected to be modified. 
These results tend to validate our numerical method with parallel gusts restriction for this cascade configuration in a uniform axial mean flow. The more realistic case of a swirling mean flow is discussed in the next section.

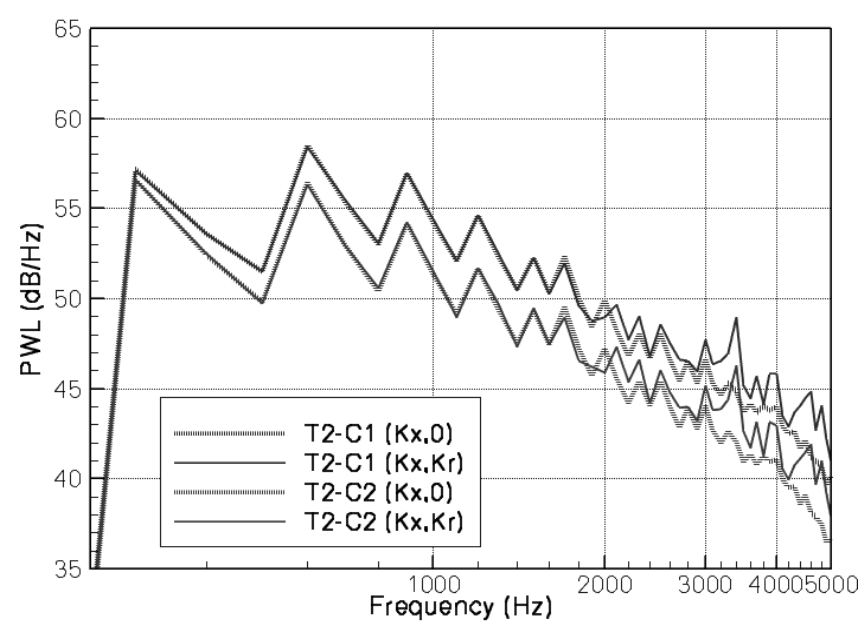

Figure 19. Comparisons of PWL spectra in the outlet duct provided by CAA+FWH computations using $\left(k_{x}, k_{r}\right)$ turbulence (solid lines) and $\left(k_{x}, 0\right)$ turbulence (dotted lines)

for T2-C1 (light) and T2-C2 (dark) cases

\section{B. Turbulence-annular cascade interaction in a swirling mean flow}

The second application case concerns a benchmark proposed by Atassi \& Vinogradov ${ }^{20}$, related to an annular grid of $V=45$ unloaded flat plates with $160 \mathrm{~mm}$ chord in a swirling mean flow. This has been also investigated by Hixon $^{32}$ but considering only a single harmonic spinning gust (tone noise), whereas we focus here to a HIT inflow condition (broadband noise). The vanes are twisted with a suited stagger angle varying along the span and adjusted so that the angle of attack remains equal to zero (local chord aligned with the streamlines). The characteristics of the annular duct are $r_{h}=0.99 \mathrm{~m}$ and $r_{t}=1.65 \mathrm{~m}$. The swirling mean flow is imposed by an axial and azimuthal component (the radial component being set to zero). The azimuthal Mach number is defined as (setting $\Omega=\Gamma=0.125$ ):

$$
M(\bar{r})=\bar{r}+\overline{\bar{r}}
$$


Using Crocco's equation and neglecting the entropy and enthalpy variations, the axial Mach number can be written as $^{34}$ :

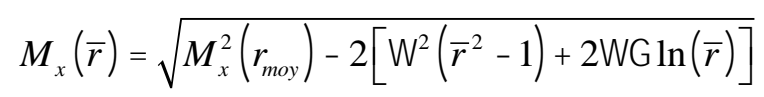

$r_{m o y}$ is equal to $1.32 \mathrm{~m}$, which corresponds to a total Mach number equal to 0.5 at this position.

The main parameters are summarized in the table 3 , in which $\bar{r}$ is the radius normalized by its mid-span value $r_{\text {moy }}$.

Table 3. Swirling mean flow and stagger angle values

\begin{tabular}{ccccc}
$r(m)$ & $\bar{r}$ & $M_{\theta}$ & $M_{x}$ & $\chi\left(^{o}\right)$ \\
\hline \hline 0.99 & 0.75 & 0.2604 & 0.4681 & 29.1 \\
1.32 & 1.00 & 0.2500 & 0.4330 & 30.0 \\
1.65 & 1.25 & 0.2563 & 0.3949 & 33.0 \\
\hline
\end{tabular}

The mean static pressure field is derived from the radial momentum equilibrium:

$$
p(\bar{r})=p_{\infty}\left[1+\left(\begin{array}{ll}
1 & 1
\end{array}\right)\left\{\left(\begin{array}{ll}
\frac{\bar{r}^{2}}{2} & 1
\end{array}\right)\left({ }^{2}+\frac{2}{\bar{r}^{2}}\right)+2 \quad \ln (\bar{r})\right\}\right]^{\prime \prime}
$$

In Eq. (6), $\gamma=1.4$, and $p=105986 \mathrm{~Pa}$. The mean density field is defined as $(\bar{r})=(p(\bar{r}) / p)^{1 /}$, and the stagger angle (set equal to the inflow angle) is deduced as $(\bar{r})=\arctan \left(M(\bar{r}) / M_{x}(\bar{r})\right)$. The radial profiles of the three fields $M_{x}(\bar{r}), M(\bar{r}), \quad(\bar{r})$ so obtained are plotted in Fig. 20.

The Cartesian coordinates $(x, y, z)$ of a point along a vane in the curvilinear frame $(,, r)$ sketched in Fig. 21 can be expressed as:

$$
\left\{\begin{aligned}
x(r) & =\cos ((r)) \\
(r) & =\frac{\sin ((r))}{r} \\
y(r) & =r \sin (\quad(r)) \\
z(r) & =r \cos (\quad(r))
\end{aligned}\right.
$$



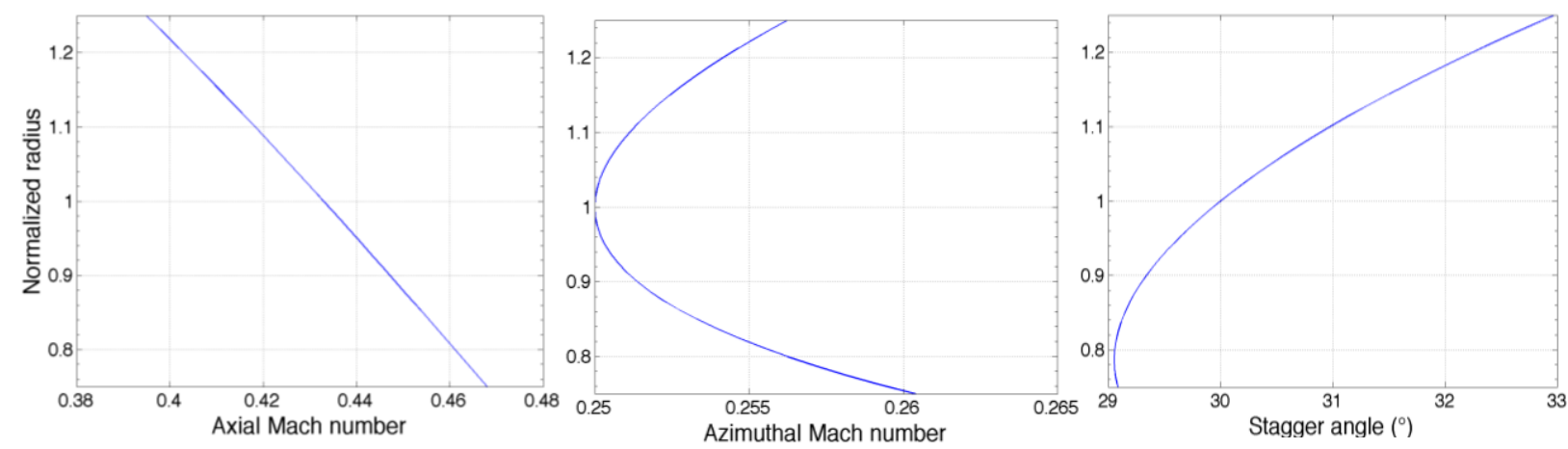

Figure 20. Radial profiles of axial (left) and azimuthal (middle) Mach number, and stagger angle (right)
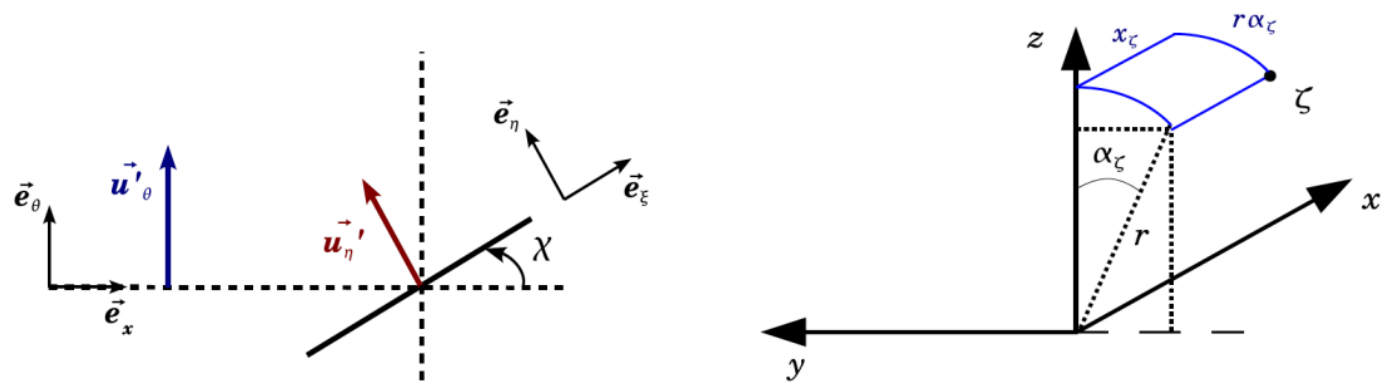

Figure 21. Local frames attached to the duct and to the vane (left) and Cartesian coordinates related to curvilinear points (right)

A $3 \mathrm{D}$ representation of the annular grid with colored stagger angle varying from $29^{\circ}$ to $33^{\circ}$ is shown in Fig. 22, and the CAA grid made of about 1.4 M points (limited to a single vane channel) is visualized in Fig. 23. The computation parameters are summarized in the table 4.

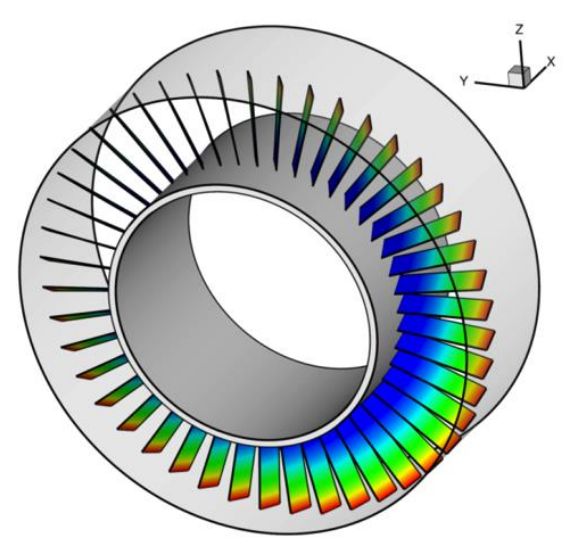

Figure 22. 3D representation (full revolution) of annular grid with colored stagger angle

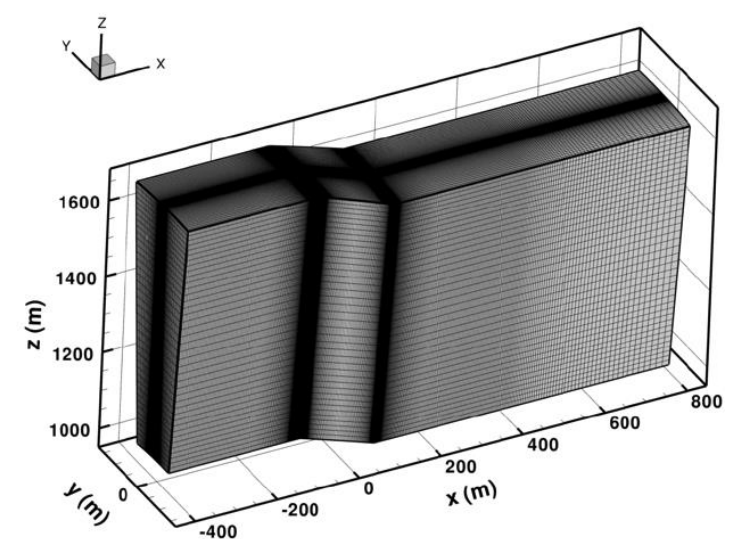

Figure 23. CAA 3D grid of single vane channel 
Table 4. CAA grid characteristics

\begin{tabular}{|c|c|c|c|c|c|}
\hline$N_{x}$ & $N_{r}$ & $N_{\theta}$ & Axial extent $(m)$ & radial extent $(m)$ & Azimuthal extent $($ rad $)$ \\
\hline 417 & 41 & 85 & $-0.4<x<0.8$ & $0.99<r<1.65$ & $-\pi / 45<\theta<\pi / 45$ \\
\hline
\end{tabular}

Due to the fact that the vane chord is not aligned with the duct axis anymore, the synthetic turbulent inflow injected into the CAA frame with Cartesian coordinates $(x, y, z)$ related to the duct cylindrical coordinates $(x, r, \quad)$ has to be expressed with respect to the local vane curvilinear coordinates $(,, r)$. Hixon $^{32}$ suggests a suited VGBC (Vortical Gust Boundary Condition) formulation at the inflow boundary in the CAA frame implemented in the BASS code from NASA Glenn Research Center, but it would require to expand the synthetic turbulence over angular modes, and so to mesh all the vanes over a $360^{\circ}$ domain. Another strategy is proposed here to still limit the CAA grid to a single vane channel. Thus, restricting again to the parallel gusts $\left(k_{r}=0\right)$ and introducing the stagger angle, the velocity disturbances of Eq. (1) are re-written as:

$$
\left\{\begin{array}{l}
u^{\prime}(x, r,, t)=2 \sum_{i=1}^{N} A_{i} \cos \left(k_{x, i} x \quad{ }_{i} t+{ }_{i}\right) \\
A_{i}=\sqrt{\frac{u_{u}\left(k_{, i} x, 0\right) k k_{r}}{\cos ((r))}}=\sqrt{\frac{u_{u}\left(k_{x, i} x \cos ((r)), 0\right) k_{x} \cos ((r)) k_{r}}{\cos ((r))}}
\end{array}\right.
$$

The wave numbers $k_{x}$ and $k$ being linked since $k=k_{x} \cos$ and $x=\cos$, the axial phase term $k_{x} x$ in Eq. (3) is actually equal to $k$ at the vane wall. One should note that by projection of $u$, a component $u$ will be also added to the upwash component $u$. However, these fluctuations are sliding along the chord and are not expected to generate any sound.

As for the previous case, the restriction to parallel gusts $\left(k_{r}=0\right.$ in Eq. (3)) in order to reduce the CPU cost is justified by the practical requirement $L / c \geq 3$. However, this simplification proposed by Amiet $^{1}$ for isolated airfoils and checked by Reboul ${ }^{23,24}$ for ducted fans is valid for non-varying inflow conditions 
along the span which is no more true here. The parallel gust restriction in the CAA then might be questionable and will be discussed below.

As defined by Atassi et al., the turbulence is modeled using the Liepmann TKE spectrum, with constant parameters $T_{u}=1.8 \%$ and $\Lambda \approx 42 \mathrm{~mm}$. Harmonic gusts are injected with a frequency spacing $\Delta f=100 \mathrm{~Hz}$ up to $f_{\max }=3300 \mathrm{~Hz}$. About 16,500 time iterations are required to simulate a complete period and a converged result is reached after 2 periods, requiring only 12 hours over 64 processors.

3D snapshot views of azimuthal velocity and pressure disturbances can be visualized in Fig. 24, left and right, respectively. As explained above, the wave fronts are almost normal to the duct axis and not to the vanes. The wave front lean traduces the radial variations of the mean flow. Wall pressure distributions provided by CAA over lower and upper vane sides are plotted in Fig. 25 (right), for several spanwise positions, and compared to the Amiet solution. A rather good agreement can be observed, despite a nonsymmetrical response with slightly higher levels predicted by the numerical simulations. It can be seen that the normalized RMS pressure levels are almost constant in the spanwise direction as highlighted by the iso-pressure contour maps plotted in Fig. 25 (left).

The PWL spectrum in the outlet duct is then calculated by coupling the CAA output data (vane surface pressure) to the code FanNoise. In Fig. 26, our CAA result (in red) is compared to Atassi's solution (in black) digitized from Ref. [20] and also to the Amiet-based prediction (in blue). Although the turbulent inflow conditions are set constant, the Amiet-based calculation is performed by splitting the duct into several radial strips (10 in the present case) in order to account for mean flow and stagger angle variations in the spanwise direction and the non compactness of the noise sources along the span. A very good agreement can be observed between Atassi and Amiet-based predictions, which tends to show that the cascade effects are negligible for this configuration. On the other hand, the present CAA solution shows significant differences, with lower PWL and particularly a steep attenuation slope beyond $1500 \mathrm{~Hz}$. This leads to an underprediction of $-8 \mathrm{~dB} / \mathrm{Hz}$ around $3000 \mathrm{~Hz}$ ). Such differences with the Amiet-based result are surprising regarding to the low discrepancies observed on the RMS wall pressure distributions (in Fig. 25 right). 

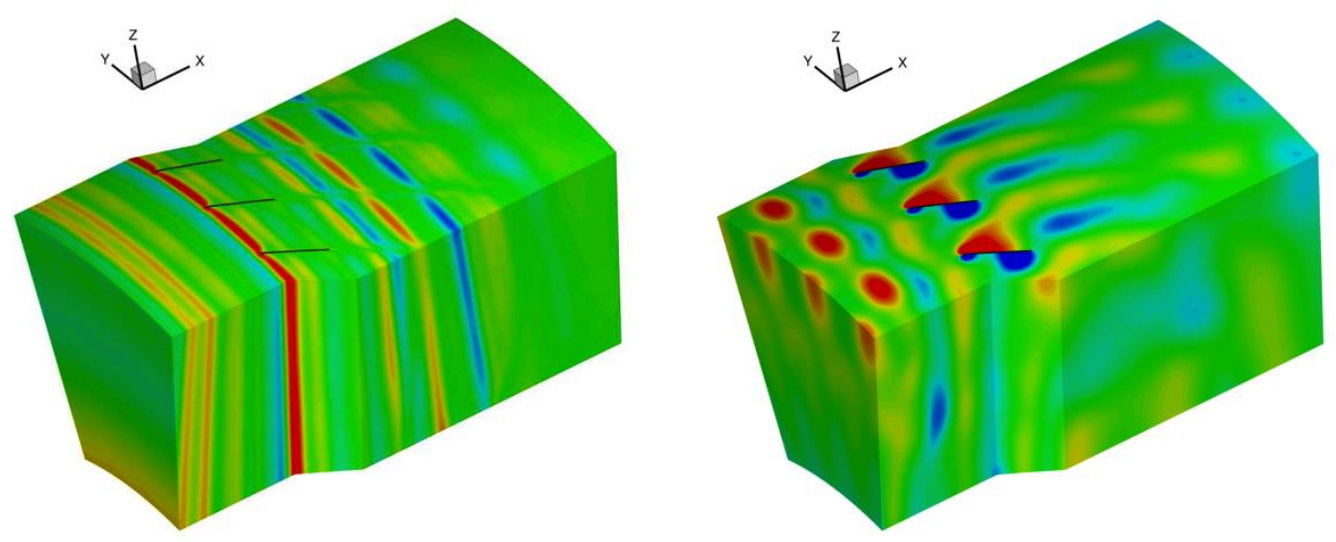

Figure 24. Snapshot of azimuthal velocity disturbances $( \pm 2 \mathrm{~m} / \mathrm{s}$, left $)$ and pressure disturbances ( $\pm 100 \mathrm{~Pa}$, right) duplicated over 3 angular sectors
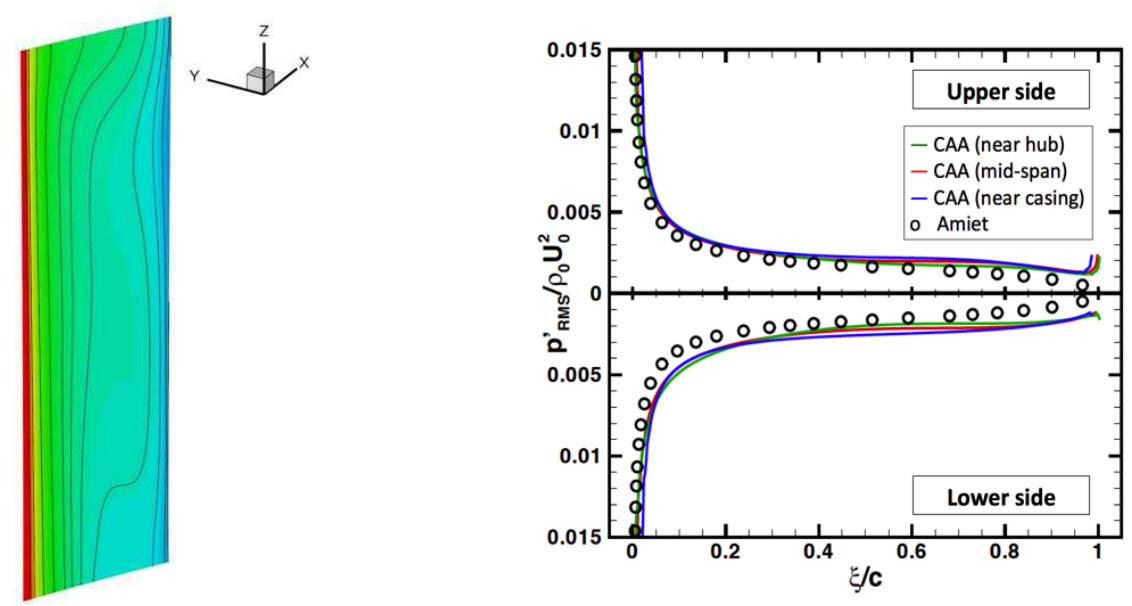

Figure 25. CAA surface RMS pressure (Pa) over the lower vane side (left) and chordwise normalized RMS pressure profiles at 3 spanwise stations compared to the Amiet-based solution (right)

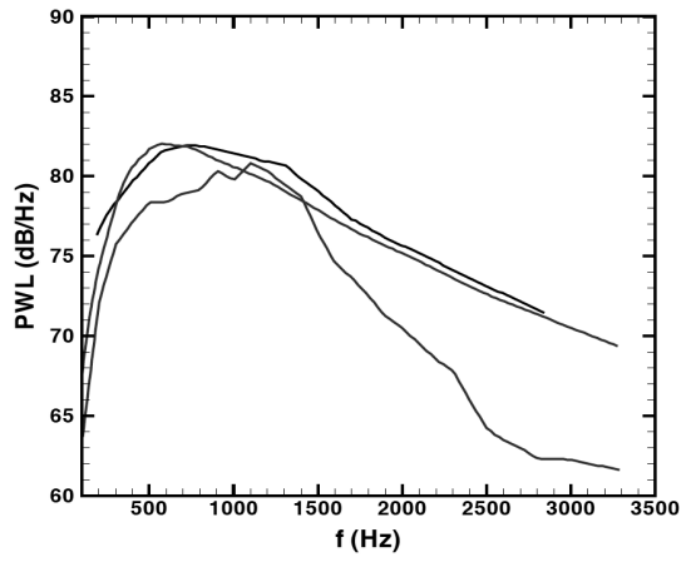

Figure 26. PWL spectra (dB/Hz) provided by $\mathrm{CAA}+\mathrm{FWH}$ (light) and compared to Amiet-based (medium) and Atassi's (black) predictions 
In order to better understand the reasons for this mismatch, wall pressure spectra have been analyzed. Typical results at two chordwise positions are presented in Fig. 27, comparing numerical pressure PSD with Amiet's theory, and in Fig. 28, showing computed phase spectra. Near the leading edge the shape and level of the computed spectra are found to be rather close to the Amiet-based predictions (in dotted lines), but important oscillations seem to appear at $10 \%$ chord. Nevertheless, the mean value of the oscillating level versus frequency, for each spanwise position, is relatively close to Amiet's reference solution. An explanation for the discrepancies shown in Fig. 26 can be inferred from Fig. 28, where significant phase variations between radial stations at $10 \%$ chord (Fig. 28 right) can be seen in the frequency range [2000-3300 Hz]. Destructive interference effects could arise from these phase shifts along the span, when integrating the wall pressure fluctuations in the FWH solver (whereas no phase shift is expected in Amiet's approach with parallel gusts). As mentioned before, the parallel gust restriction in the CAA might not be suited to realistic configurations with mean swirling flows.

To check this point, a FWH calculation has been run again by discarding the phase information along the span (source correlation is only considered in the chordwise direction). The numerical prediction obtained by this way is plotted in Fig. 29 and compared to the previous solutions, showing an increase of the PWL up to $4 \mathrm{~dB} / \mathrm{Hz}$ and leading to a better agreement with Atassi's results. This tends to confirm our interpretation and provides some limits of our present numerical method when discarding the oblique gusts for this second turbulence-cascade application case.
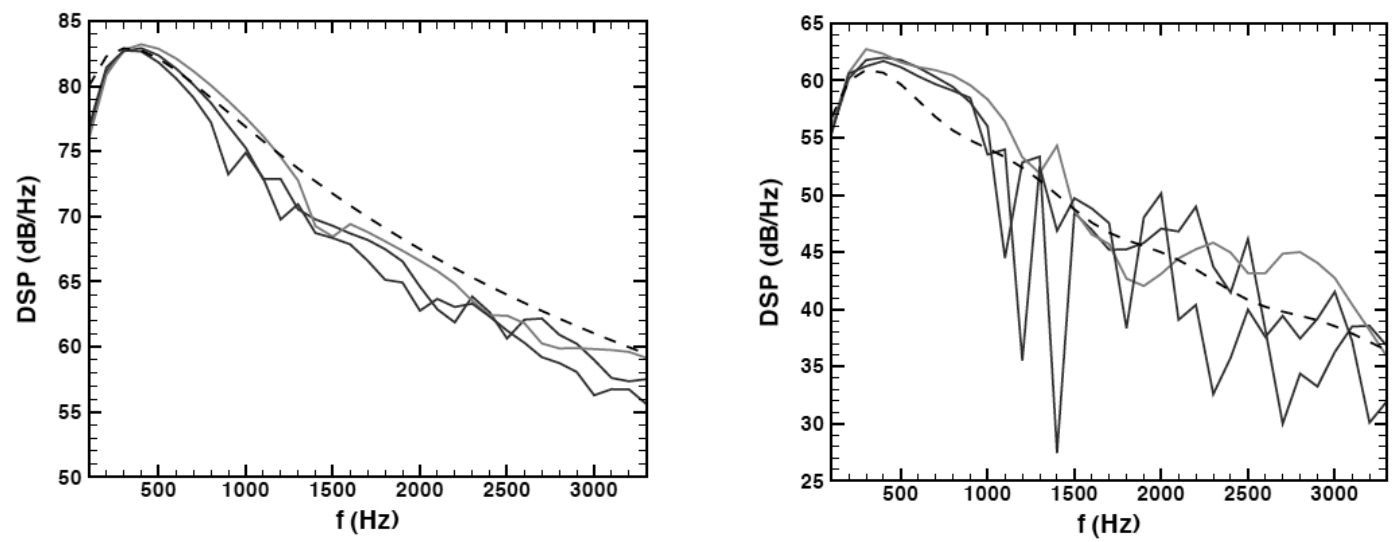

Figure 27. CAA wall pressure PSD $(\mathrm{dB} / \mathrm{Hz})$ on vane pressure side (hub/light, mid-span/medium, casing/dark) compared to Amiet-based solution (dotted line) at $\quad / c \approx 0$ (left) and $\quad / c=0.1 c$ (right). 

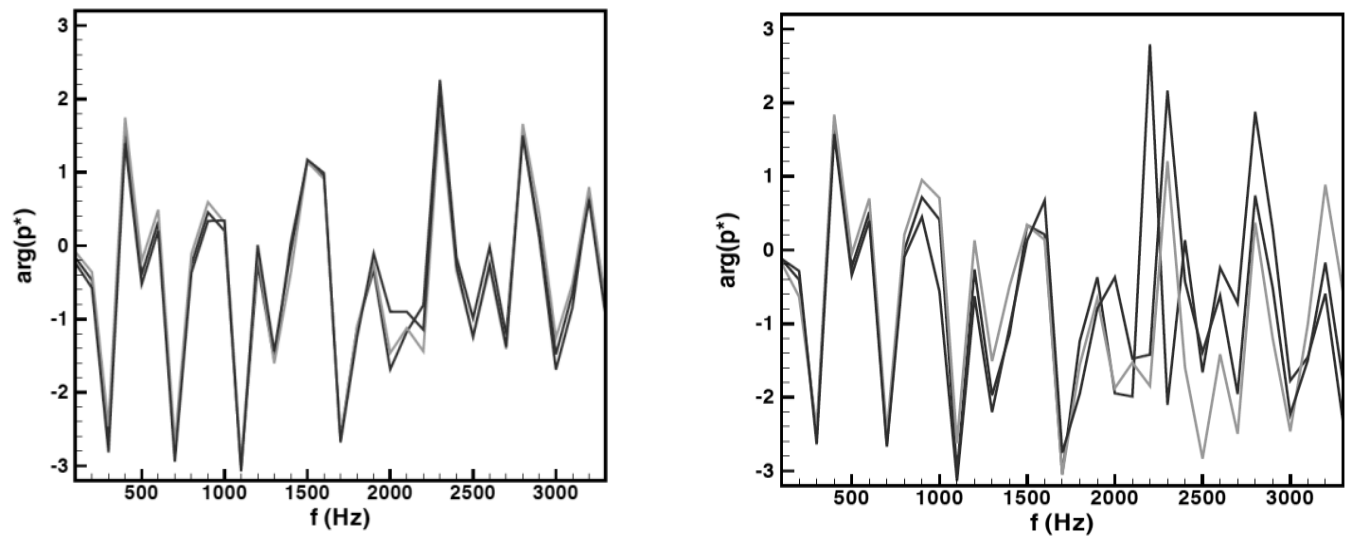

Figure 28. CAA wall pressure PSD phase (radians) on vane pressure side (hub/light, mid-span/medium, casing/dark) at $\quad / c \approx 0$ (left) and $/ c=0.1 c$ (right)

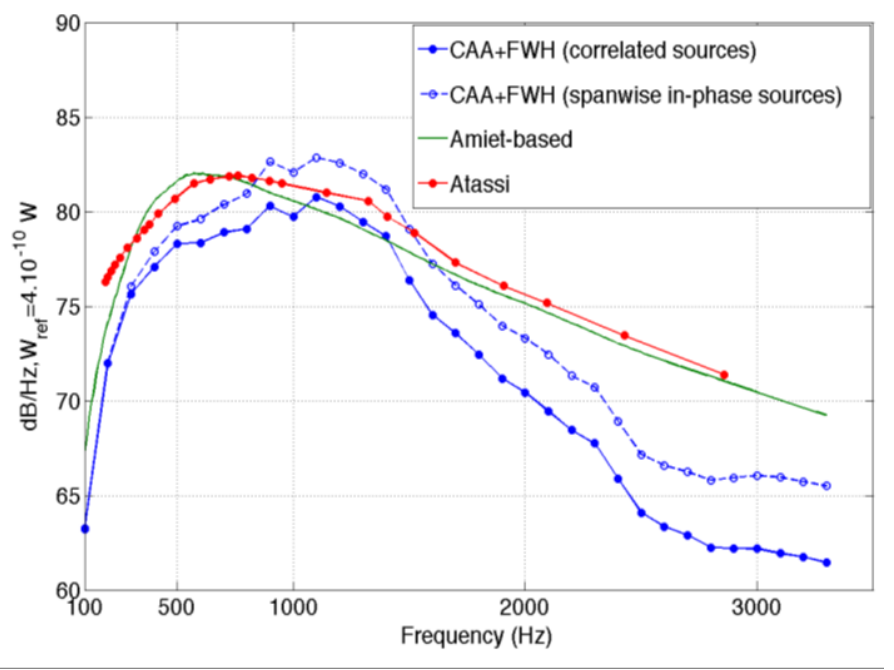

Figure 29. $\mathrm{PWL}$ spectra $(\mathrm{dB} / \mathrm{Hz})$ provided by $\mathrm{CAA}+\mathrm{FWH}$ compared to Amiet-based and Atassi's solutions

As done in section IV.A, in order to check the accuracy of our numerical simulations and to confirm the above suggestions, extended computations have been performed using the complete $\left(k_{x}, k_{r}\right)$ turbulence spectrum in Eq. (8) and applying a double sum over axial and radial wave numbers as written in Eq. (1). The same CAA grid with identical computation parameters have been used. Moreover, to better estimate the PWL deviations related to statistical errors, three different sets of random phases have been used when generating the synthetic turbulence, leading to three independent runs and simulation results. 
Referring to Shannon, up to 21 radial modes ( $M=10$ in Eq. (1)) can be generated here, and convergence is achieved after 2 periods requiring about 10 hours over 64 processors (comparable to the previous case).

First of all, computation results have been focused on wall pressure analyzes over the vanes. Typical solutions near the leading edge are presented in Fig. 30, in terms of pressure PSD (Fig. 30 (a)) with associated phase spectra (Fig. 30 (b)), for 3 spanwise positions (10\% span in green, $50 \%$ span in red, and 90\% span in blue, as in Figs. 27 and 28). As expected, the pressure PSD shapes are much more oscillating (compared to those in Fig. 27 left) with higher levels too. Contrary to Fig. 28 left, phase spectra exhibit strong variations along the span due to the presence of the oblique gusts that should give rise to more relevant decorrelations effects. PWL spectra in the outlet duct provided by the coupling with FanNoise code for the three different random phase sets (3 different CAA runs) are compared in Fig. 31. Despite of the oscillations, the spectra display quite similar shapes so that a smoothing issued from a quadratic average of the levels would converge to very close solutions. To finish, the outlet duct PWL spectrum provided by CAA using $\left(k_{x}, k_{r}\right)$ turbulence spectrum model (and phase set 3 ) is compared to the one previously obtained (using $\left(k_{x}, 0\right)$ ) and to Atassi solution in Fig. 32. The numerical prediction is found to be clearly improved, showing a much better agreement with Atassi's spectrum, with a better assessment of the attenuation slope, despite slightly overpredicted levels (no more than $3 \mathrm{~dB}$ ). This is a very promising result that tends to validate the present method, and suggests to use the complete 2-wave number turbulence model for rotor-stator applications. 


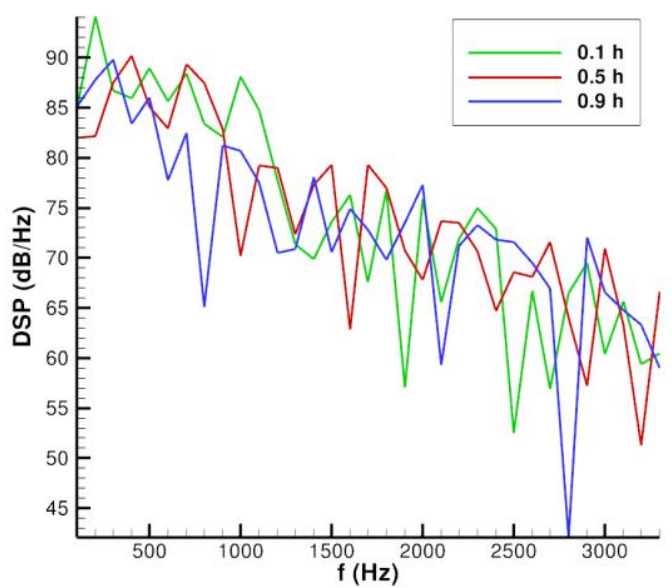

a) Pressure PSD $(\mathrm{dB} / \mathrm{Hz})$

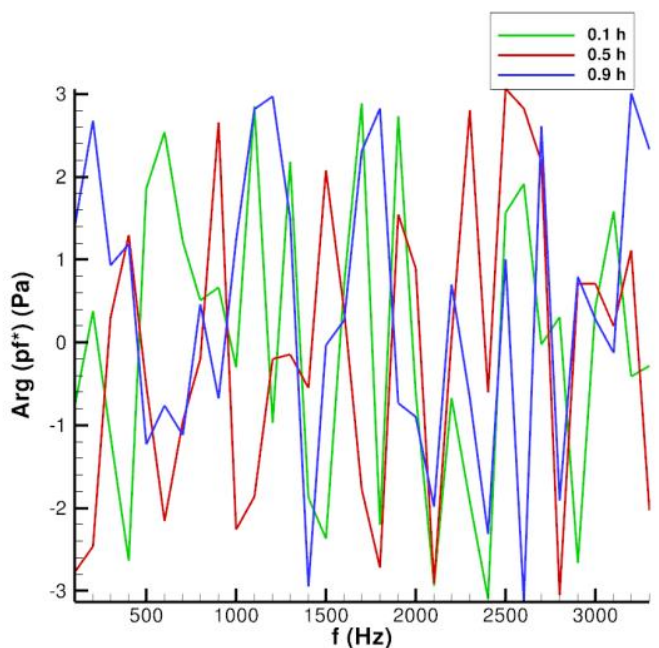

b) Phase spectra (radians)

Figure 30. CAA predictions using complete synthetic turbulence with radial wave numbers:

(a) Wall pressure PSD, and (b) phase spectra (in radians), at three spanwise positions

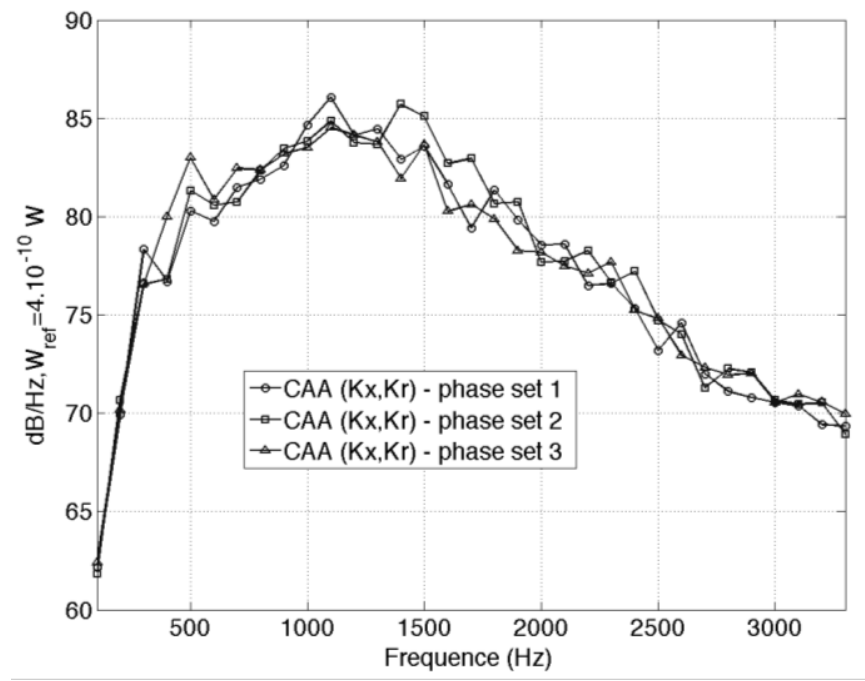

Figure 31. Outlet duct PWL spectra $(\mathrm{dB} / \mathrm{Hz})$ issued from CAA with $\left(k_{x}, k_{r}\right)$ turbulence spectrum using different sets of random phases 


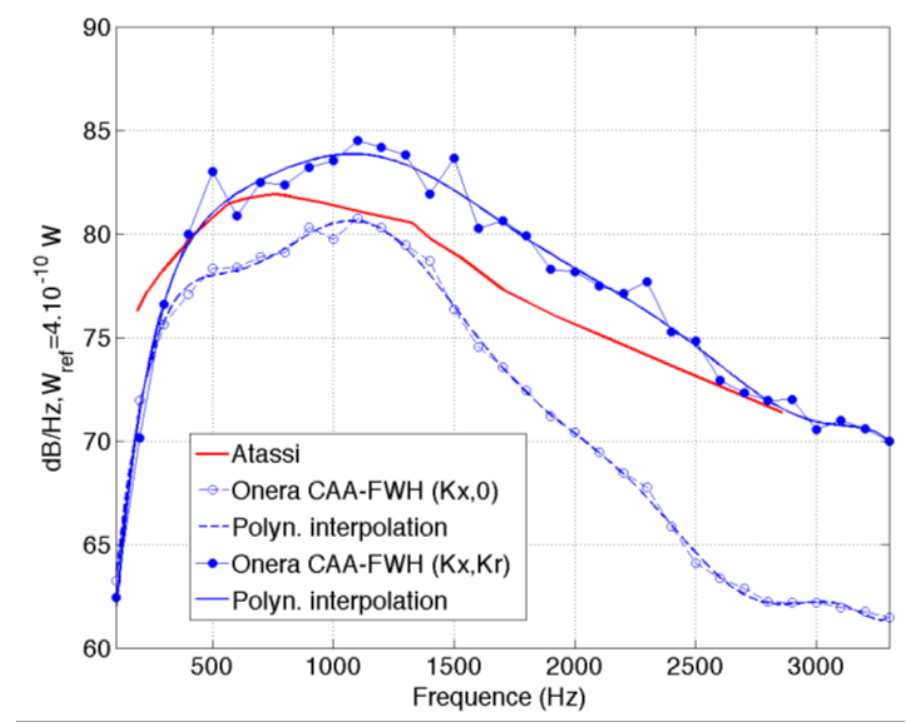

Figure 32. Outlet duct PWL spectra $(\mathrm{dB} / \mathrm{Hz})$ provided by $\mathrm{CAA}+\mathrm{FWH}$ using $\left(k_{x}, 0\right)$ and $\left(k_{x}, k_{r}\right)$ turbulence spectra and compared to Atassi's solution

\section{Conclusions and Future Work}

A hybrid methodology based on a 3D CAA/Euler solver coupled to a stochastic model aiming at generating a synthetic turbulent inflow has been presented in this paper. Suited Tam's boundary conditions have been implemented into the code to ensure a non-reflecting injection of velocity disturbances, and have been associated to a sponge zone at the exit for outgoing acoustic/hydrodynamic modes. Simulations have been conducted on annular flat-plate cascade configurations with prescribed inflow disturbances impinging the flat-plate vanes. Both uniform and swirling mean flow cases have been investigated, and the numerical predictions have been successfully compared to semi-analytical solutions and to experimental data (when available). Sound propagation simulations in the duct are practically achieved by chaining the CAA code to a FWH solver for broadband noise calculations.

The present method has been first validated against an harmonic CAA benchmark proposed by NASA, showing an excellent agreement. The use of a 2-wave numbers (streamwise and spanwise) turbulence spectrum resulting from an integration of the 3-wave numbers spectrum over the azimuthal direction has been suggested in order to limit the computation domain to a single vane channel. Furthermore, by 
analogy with Amiet theory, synthetic turbulence can be restricted to parallel gusts (setting the spanwise wave number equal to zero). This allows us to considerably reduce the CPU costs. Our predictions, applied to two tested cascades immersed in a turbulence convected by a purely axial mean flow, are found to be close to semi-analytical solutions based on lifting surface method and to the measurements. It has been verified that the addition of oblique gusts (spanwise wave numbers) lead to almost the same PWL spectra when the mean flow is purely axial. A first application to a more realistic swirling mean flow case (involving a radial evolution of the convection flow and stagger angle) has been investigated then, showing that the spanwise wave number contribution has to be included into the CAA simulations in order to capture realistic de-correlation effects along the span. By this way, the present numerical predictions were found to be in a rather good agreement with Atassi reference solution.

The next step will be to apply the method to a turbofan stage model configuration like the SDT from NASA Glenn ${ }^{35}$, recently benchmarked using semi-analytical ${ }^{19}$ and numerical ${ }^{7,27}$ methods, and to adjust the turbulent inflow characteristics from hot-wire measurements or from a RANS computation data.

\section{Acknowledgements}

This work was partly conducted in the framework of the LabEx CeLyA ("Centre Lyonnais d'Acoustique") of Université de Lyon, operated by the French National Research Agency. Authors would like to thank Julissa Grondin (Master's student at ECL) for the updated CAA simulations with oblique gusts realized during her internship at ONERA.

\section{References}

${ }^{1}$ Amiet, R. K., Acoustic radiation from an airfoil in turbulent stream, J. Sound and Vib., 41(4), pp. 407-420, 1975.

${ }^{2}$ Hanson, D., Broadband noise of fans. with unsteady coupling theory to account for rotor and stator reflection/transmission effects, NASA Contractor Report 211136, 2001. 
${ }^{3}$ Riou, J., Léwy, S. \& Heib, S., Large eddy simulation for predicting rotor-stator broadband interaction fan noise, Inter-noise2007 Conference, 2007.

${ }^{4}$ Greschner, B. \& Thiele, F., Wall modeled LES simulation of rotor-stator-cascade broadband noise, 17th AIAA/CEAS Aeroacoustics Conference, AIAA Paper 2011-2873, 2011.

${ }^{5}$ Laborderie, J., Moreau, S. \& Berry, A., Compressor Stage Broadband Noise Prediction using a

Large-Eddy Simulation and Comparisons with a Cascade Response Model, AIAA Paper 2013-2042, 2013.

${ }^{6} \mathrm{Li}$, Q., Peake, N., and Savill, M., Large Eddy Simulations for Fan-OGV Broadband Noise Prediction, AIAA Paper 2008-2843, 2008.

${ }^{7}$ Mann, A., Perot, F., Kim, M.-S., Casalino, D. \& Fares, E., 2012, Advanced noise control fan direct aeroacoustics predictions using a Lattice-Boltzmann method, 18th AIAA/CEAS Aeroacoustics Conference, AIAA Paper 2012-2287, 2012.

${ }^{8}$ Clair, V., Polacsek, C., Le Garrec, T., Reboul, G., Gruber, M. \& Joseph, P., Experimental and Numerical Investigation of Turbulence-Airfoil Noise Reduction Using Wavy Edges, AIAA Journal, Vol. 51, No. 11, pp. 2695-2713, 2013.

${ }^{9}$ Dieste, M., and Gabard, G., Random particle methods applied to broadband fan interaction noise, Journal of Computational Physics, 231, pp. 8133-8151, 2012.

${ }^{10}$ Wohlbrandt, A., M., Guerin, S., Ewert, R., Simultaneous Computation of Surface and Volume Sources for Fan Broadband Noise with the Random-Particle-Mesh Method, 19th AIAA/CEAS Conference, AIAA Paper 2013-2119.

${ }^{11}$ Clair, V., Polacsek, C., Le Garrec, T., Jacob, M., Numerical Predictions of Turbulence-Cascade Interaction Noise Using CAA with a Stochastic Model, 20th AIAA/CEAS Aeroacoustics Conference, AIAA Paper 2014-2453, 2014.

${ }^{12}$ Redonnet, S., Manoha, E., and Sagaut, P., Numerical Simulations of Propagation of Small Perturbations Interacting with Flows and Solid Bodies, 7th AIAA/CEAS Aeroacoustics Conference, Paper 2001-0222, 2001. 
${ }^{13}$ Redonnet, S., Desquesnes, G., Manoha, E., and Parzini, C., Numerical study of acoustic installation effects with a computational aeroacoustics method, AIAA Journal, 48(5), 2010.

${ }^{14}$ Tam, C. K. W., Advances in Numerical Boundary Conditions for Computational Aeroacoustics, Journal of Computational Acoustics, Vol. 6, No. 4, pp. 377-402, 1998.

${ }^{15}$ Namba, M. \& Schulten, J., Third computational aeroacoustics (CAA) workshop on benchmark problems: Category 4 - fan stator with harmonic excitation by rotor wake, NASA Technical Memorandum, 2000-209790, pp. 73-85, 2000.

${ }^{16}$ Namba, M., Three-dimensional analysis of blade force and sound generation for an annular cascade in distorted flows, J. Sound and Vib., 50, p. 479-508, 1977.

${ }^{17}$ Schulten, B., Sound generated by rotor wakes interacting with a leaned vane stator, AIAA Journal, 20(10), p. 1352-1358, 1982.

${ }^{18}$ Posson, H. \& Roger, M., Experimental validation of a cascade response function for fan broadband noise predictions, AIAA Journal, Vol. 49, No. 9, p. 1907-1918, 2011.

${ }^{19}$ Envia, E., and Coupland J., AA-39 Panel Session: Fan Broadband Noise Prediction, www.oai.org/aeroacoustics/FBNWorkshop/, 20th AIAA/CEAS Aeroacoustics Conference, 2014.

${ }^{20}$ Atassi, H. \& Vinogradov, I., Modeling broadband fan noise and comparison with experiments, 13th AIAA/CEAS Aeroacoustics Conference, AIAA Paper 2007-3691, 2007.

${ }^{21}$ Atassi, H., Ali, A., Atassi, O. \& Vinogradov, I., Scattering of incident disturbances by an annular cascade in a swirling flow, Journal of Fluid Mechanics, 499, p. 111-138, 2004.

${ }^{22}$ Goldstein M.-E., Aeroacoustics, McGraw-Hill, New-York (USA), 1976.

${ }^{23}$ Reboul, G., Polacsek, C., Lewy, S. \& Heib, S., Ducted-fan broadband noise simulations using unsteady or averaged data, Inter-noise2008 Conference, 2008.

${ }^{24}$ Reboul, G., Modélisation du bruit à large bande de soufflante de turboréacteur [In French], PhD Thesis, Ecole Centrale de Lyon, 2010.

${ }^{25}$ Zhang, W., Wang, X. \& Sun, X., A broadband noise model for turbulencelannular cascade interaction, 19th AIAA/CEAS Aeroacoustics Conference, AIAA Paper 2013-2243, 2013. 
${ }^{26}$ Bogey, C. \& Bailly, C., Three-dimensional non-reflective boundary conditions for acoustic simulations: far-field formulation and validation test cases, Acta Acustica, 88, p. 463-471, 2002.

${ }^{27}$ Sescu, A., Towards Computational Aeroacoustics Prediction of Realistic Turbofan Noise Using Synthetic Turbulence Modeling, PhD Thesis, University of Toledo (USA), 2011.

${ }^{28}$ Kraichnan, R., Diffusion by a random velocity field, Physics of Fluids, 13(1), p. 22-31, 1970.

${ }^{29}$ Salem Said, A.-H., Large Eddy Simulation of Shear-Free Interaction of Homogeneous Turbulence with a Flat-Plate Cascade, PhD Thesis, Virginia Polytechnic Institute and State University, 2007.

${ }^{30}$ Ewert, R, Broadband slat noise prediction based on CAA and stochastic sound sources from a random particle mesh (RPM) method, Computers and Fluids, 37, pp. 369-387, 2008.

${ }^{31}$ Casper, J. \& Farassat, F., Broadband noise predictions based on a new aeroacoustic formulation, 40th Aerospace Sciences Meeting and Exhibit, AIAA Paper 2002-0802, 2002.

${ }^{32}$ Hixon, R., Sescu, A., Sawyer, S., Vortical gust boundary condition for realistic rotor wake/stator interaction noise prediction using computational aeroacoustics, J. Sound Vib., 330, pp. 3801-3817, 2011.

${ }^{33}$ Tyler, M., Sofrin, T. G, Axial Flow Compressor Noise Studies, Society of automotive engineers transactions, Vol. 70, pp. 309-332, 1962.

${ }^{34}$ Golubev, V. \& Atassi, H., Unsteady Swirling Flows in Annular Cascades, Part 1: Evolution of Incident Disturbances, AIAA Journal, 38(7), p. 1148-1149, 2000.

${ }^{35}$ Envia E., Tweedt D.-L., Woodward R.-P., Elliott D.-M., Fite E.-B., Hughes C.-E., Podboy G.-G., Sutliff D.-L., An assessment of current fan noise prediction capability, in 14th AIAA/CEAS Aeroacoustics Conference, AIAA-2008-2991, 2008.

${ }^{36}$ Rienstra, S. W., Acoustic Radiation from a Semi-Infinite Annular Duct in Uniform Subsonic Mean Flow, J. Sound Vib., 94(2), 1984. 


\section{Appendix}

\section{A. Amiet-based prediction of rotor-stator interaction broadband noise}

Here after, a short description of the formulation developed in Ref [24] is presented. The model is based on the prediction of the surface pressure spectral density over the stator (cascade) vane using an aeroacoustic transfert function based on Amiet's theory ${ }^{1}$ (isolated airfoil response assimilated to a flatplate) and coupled to the FWH formulation (loading noise term) extended by Goldstein ${ }^{22}$ using a Green's function valid for an annular duct and a uniform mean flow. We use a bidimensional form of Amiet-based response by only considering the parrallel gusts. This hypothesis is justified for large aspect ratio $(L / c \geq 3)$ and allows very fast predictions since no integration over the radial wavenumbers is necessary. Hence, we can easily apply a strip theory that consists in splitting the incoming turbulence characteristics in several slices along the span and performing an incoherent sum of each slice contribution. Sources are distributed over each strip center, so that the formulation is partially non compact.

The flat-plate attached coordinate system $(,, r)$ in the $x$-axis duct frame are sketched in Fig. A.1.

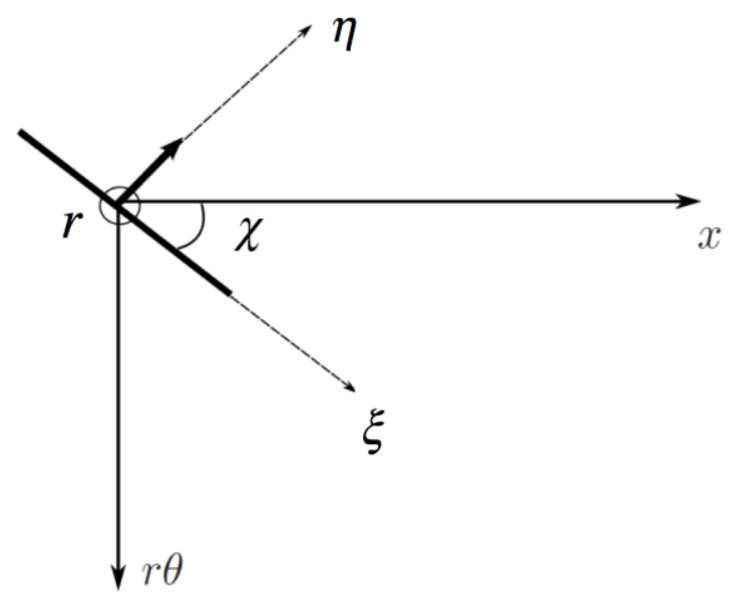

Figure. A.1. Flat-plate vane coordinates in the annular duct [24]

The classical expression of PWL spectrum, $S_{w w}(f)$, writes:

$$
S_{w w}^{ \pm}(f)=\sum_{m=m_{\max }}^{m_{\max }} \sum_{n=1}^{n_{\max }} \frac{{ }_{m n} r_{t}^{2}}{{ }_{0} c_{0}} \frac{K\left({ }^{2} k_{m n}^{ \pm}+M_{x} K\right)}{\left(K \quad M_{x} k_{m n}^{ \pm}\right)^{2}} E\left[\left|A_{m n}^{ \pm}(f)\right|^{2}\right]
$$


\pm denotes downstream (+) and upstream (-) propagation, $K$ is the total acoustic wave number, $k_{m n}^{ \pm}$is the axial wavenumber of the mode $(m, n),{ }^{2}=1 \quad M_{x}^{2}$ with $M_{x}$ is the axial Mach number in the duct, and $\Gamma_{m n}$ is the normalization factor (over the duct cross-section) of the orthogonal eigenfunctions. $E\left[\left|A_{m n}^{ \pm}(f)\right|^{2}\right]$ is the ensemble average of the duct mode amplitude $A_{m n}$. In the present model, this last quantity is given for one strip at spanwise station $r=r_{s}$, by:

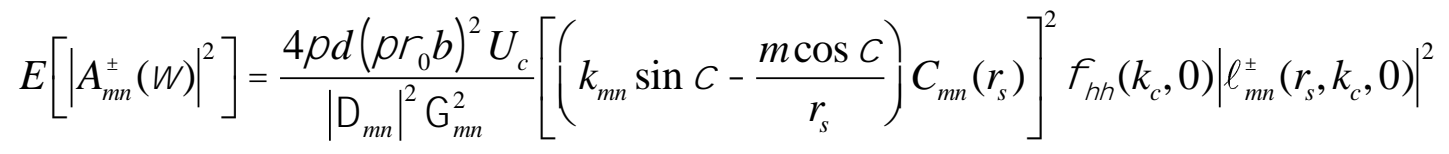

In the last equation, $d$ is half the size of the considered strip, $b$ is the half-chord and the stagger angle. The incoming turbulence is supposed to be homogeneous, uniform azimutally and frozen with a convection velocity, $U_{c}$ and a convection wavenumber, $k_{c}=\frac{}{U_{c}}$. The fluctuations of the upwash velocity component (normal to the chord) are described by the upwash velocity spectrum.$\Delta_{m n}$ is the cut-off ratio of the mode $(m, n)$ and $C_{m n}$ is the radial eigenfunction (suited normalized functions proposed by Rienstra ${ }^{36}$ are adopted, so that $\Gamma_{m n}=2 \pi$ ). Finally, $\ell_{m n}$ is an aeroacoustic modal transfert function, related to Amiet's response function, $g$, in equation (A3).

$$
\ell_{m n}^{ \pm}\left(r_{s}, k_{c}, k_{r}\right)=\frac{1}{b}_{b}^{b} g\left(, k_{c}, k_{r}\right) e^{i \stackrel{ \pm}{m n}\left(r_{s}\right)} d
$$

${ }_{m n}$ is a phase term related to the Green's function at source position ( $s$ indice on axial and angular cylindrical coordinates) and defined by:

$$
{ }_{m n}^{ \pm}\left(r_{s}\right)=m_{s}+k_{m n}^{ \pm} x_{s}
$$


Following Amiet's methodology, it is possible to split $\ell_{m n}$ in two parts: a main term, $\ell_{m n}^{1}$, corresponding to the contribution of the leading edge and a correction term, $\ell_{m n}^{2}$, induces by the trainling edge. For supercritical gusts $\left(k_{r} \leq \frac{k_{c} M_{c}}{}\right)$ :

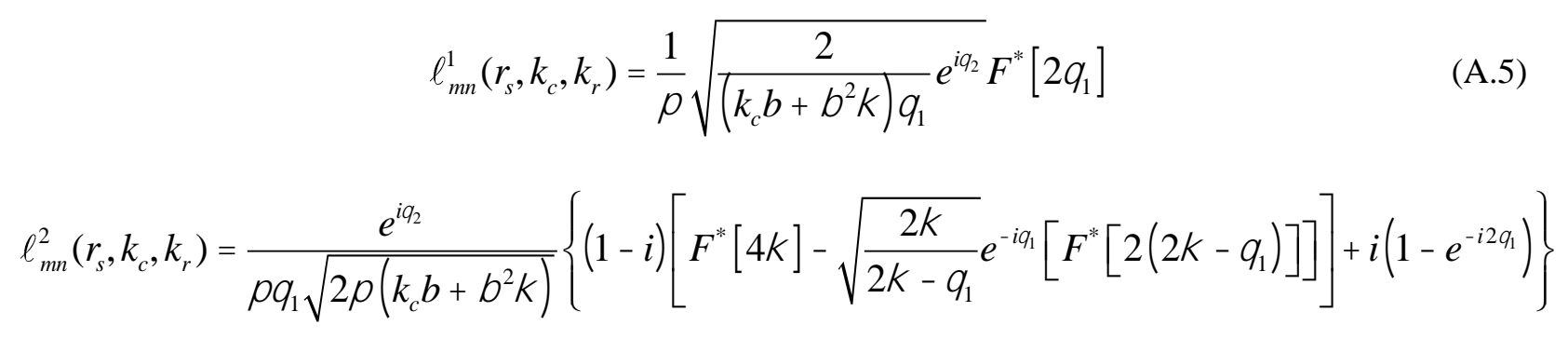

where ${ }^{2}=2 \frac{k_{r}^{2} b}{2},=\frac{M_{c} k_{c} b}{2},{ }_{1}=M_{c} \quad b_{m n}\left(r_{s}\right),{ }_{2}=b_{m n}\left(r_{s}\right) \quad / 4$ and $F^{*}$ is the conjugate of the complex Fresnel integral. Expressions for subcritical gust are available in Ref. [24], but are not practically required since spanwise wavenumbers $k_{r}$ are discarded. 